\title{
A finite line source model with Cauchy-type top boundary conditions for simulating near surface effects on borehole heat exchangers
}

\author{
Jaime A. Rivera ${ }^{\mathrm{a},{ }^{*}}$, Philipp Blum ${ }^{\mathrm{b}}$, Peter Bayer ${ }^{\mathrm{a}}$ \\ ${ }^{a}$ ETH Zurich, Department of Earth Sciences, Sonneggstrasse 5, 8092 Zurich, Switzerland \\ ${ }^{\mathrm{b}}$ Karlsruhe Institute of Technology (KIT), Institute for Applied Geosciences (AGW), Kaiserstraße 12, \\ 76131 Karlsruhe, Germany \\ ${ }^{*}$ Corresponding author. Tel.: +41-44-6332594, fax: +41-44-6331108. E-mail address: \\ jaime.rivera@erdw.ethz.ch
}

Keywords: ground heat exchange; ground surface temperature; shallow geothermal energy; finite line source; ground source heat pump.

\begin{abstract}
Borehole heat exchangers (BHEs) are the most common shallow geothermal applications. By approximating the BHE as a line source, semi-analytical models can describe the heat exchange within the ground. These models though always assume prescribed temperature at the ground surface. This work presents a formulation which expands existing finite line source models by implementing a more general Cauchy-type top boundary condition and in this way, a better estimation of the heat fluxes at the ground surface. The new formulation is numerically verified and examined in a dimensionless analysis. It is demonstrated that the discrepancy to prescribed temperature settings is significant near to the ground surface, and it propagates deeper when groundwater flow is absent and when strong decoupling between the thermal regimes interacting at the land surface is assumed. The new approach shows to be suited especially for short BHEs, both for more flexible and accurate prediction of the ground thermal regime as well as for long-term analysis of technological performance.
\end{abstract}




\section{Introduction}

The utilization of low-enthalpy geothermal energy focuses on the shallow subsurface of some hundreds of meters depth. Most commonly, so-called ground heat exchangers or borehole heat exchangers (BHEs) are installed. These exchange heat with the ground by circulating a fluid through tubes installed in vertical boreholes. BHEs are usually connected to heat pumps defining the so-called ground source heat pump system (GSHPs). During the last decade, the number of BHEs has significantly grown, especially in cities of central and northern Europe, the USA and China [1, 2]. In 2015, worldwide annual utilization of GSHPs is estimated to reach 325 PJ [3].

With their number and density growing, there is also rising interest in improved simulation techniques to characterize and predict the thermal response in the ground. The most elementary simulation techniques are based on Kelvin's line source [4, 5]. For example, the semi-analytical, infinite line-source solution is suitable for modelling seasonal energy exchange. It treats the ground as an initially isothermal, homogeneous medium, where heat is transported by conduction only. The BHE is approximated as a vertical line of infinite length embedded in the ground. Depending on the net thermal load on the system (net balance between heating and cooling loads), the line source creates a radial and expanding temperature gradient during system operation. After a typical operational life span (between 30 and 50 years), the extent can be up to tens of meters. On the long term, this may also affect neighbouring geothermal systems [6-10].

By approximating the ground as infinite conductive system, any further processes and boundary conditions are neglected. These, however, have often shown to be crucial, and thus advanced line source solutions have been proposed. Recent advancements in analytical BHE modelling focus on improved expressions for more efficient computer-based implementation, short term simulation and high time-resolution of operation with discontinuous heat extraction or injection [11-14]. Li and Lai $[15,16]$ develop techniques to deal with anisotropy and heterogeneity of the surrounding medium with analytical models. One process is advective heat flux stimulated by horizontal groundwater flow. In fact, many BHEs are installed in dynamic aquifers, so that even in a conduction-dominated environment, advection may have a remarkable influence on the evolution of ground temperatures around BHEs [17]. Thermal anomalies are deformed to heat or cold plumes elongated in downstream groundwater flow direction [18, 19]. Sutton et al. [20] and Diao et al. [21] showed how to include horizontal advection in the so-called moving line source [4, 22]. Molina-Giraldo et al. [23] included mechanical dispersion, and Molina-Giraldo et al. [24] introduced a moving finite line source (MFLS). Even if BHEs are installed in long 
boreholes, the simulation as finite lines is more accurate and especially axial effects at the borehole toe can be considered [25-28]. Thermal anomalies would be overestimated when modelling a BHE as infinite, whereas the calculation error increases the shorter the BHE is.

Existing BHE models implement the top boundary of the line source as Dirichlet-type ( $1^{\text {st }}-$ kind $)$ boundary condition, either as fixed $[6,21,24]$ or time-dependent temperature [29, 30]. Even if formulations with $1^{\text {st }}$-kind condition can consider variations in space and time, they represent general simplifications of the situation in the field. For example, when the ground surface temperature is assumed to be equal to the atmospheric temperature, the isolating effect of snow cover is ignored [31,32]. Generally, the surface air temperature (SAT) is more accessible and better monitored than the ground surface temperature (GST). Existing models often take the SAT as an approximation for the GST neglecting the different behaviours and coupling of both temperature fields [33]. As an alternative, the Cauchy-type or $3^{\text {rd }}$-kind boundary condition assumes a linear heat transfer rate between the subsurface's upper layer and the atmosphere. This boundary formulation represents, in a more realistic manner, the thermal regime near to the ground and hence constitutes a more general approach for this boundary-value problem [4, 34].

Formulations with Cauchy-type boundary conditions are common in land-surface models (e.g. $[35,36])$. Analytical models have been developed to study land-atmosphere processes but without including the coupled effect of shallow geothermal systems (e.g. [37, 38]). Cauchy-type boundary conditions are also applied for simulating shallow geothermal applications such as aquifer thermal storage systems ATES, energy piles and very-shallow helical ground heat exchangers [39-43]. However, for these applications, even simple problems are exclusively solved by numerical techniques.

In this work proposes an alternative analytical model framework that can handle Cauchy-type boundaries for analysing the thermal effects of BHEs in the ground. It is fast, can incorporate horizontal groundwater flow and simulate transient thermal conditions. The model is numerically verified and sensitivity analyses are provided to reveal the role of conduction, advection and boundary settings. 


\section{Methodology}

Figure 1 schematically shows a vertical profile with a BHE implemented in a three-dimensional (3-D) semi-infinite space. The BHE is approximated as a line source with constant heat extraction/injection rate $q$ and length $H$. It is also assumed constant, isotropic and homogeneous flow and transport properties of the porous medium (e.g. [21, 44, 45]). This homogenization strategy has shown to be a good approximation specially in conduction-dominated systems ([28, 46]). For the system depicted in Figure 1, the transient temperature distribution is given by the heat transport equation (e.g. $[4,47])$ :

$$
\frac{\partial T}{\partial t}+\boldsymbol{v} \cdot \nabla T=\nabla \cdot(a T)
$$

where $v$ is the effective thermal velocity vector with magnitude $v=D \frac{c_{w}}{c}$. The Darcy flux, $D$, is assumed uniform and steady. This may be a strong assumption depending on the specific case. However, it is not expected that $\boldsymbol{v}$ plays an important role in the temperature response close to the ground surface. In other applications characterized by complex hydrogeological conditions, numerical models are probably the only way around (e.g. $[48,49])$. In Eq. (1), the parameter $a$ represents the effective thermal diffusivity given by $a=\frac{\lambda}{c}$, where $\lambda$ and $c$ are the effective conductivity and effective volumetric heat capacity of the porous medium, respectively.

A Neumann-type boundary condition (BC) represents the BHE by $([4,5])$

$$
\lim _{r_{b} \rightarrow 0} 2 \pi r_{b} \lambda \frac{\partial T}{\partial r_{b}}=-q, \quad 0 \leq z \leq H
$$

where $r_{b}$ is the borehole radius. A Cauchy-type BC is imposed at the ground surface to account for a linear heat transfer between the subsurface and the atmosphere. In this BC, the heat flux is proportional to the difference between the ground surface temperature (GST) $T$ and the temperature of the medium above the ground $T_{S}$ (e.g. SAT). This temperature as well as the initial temperature field are equal to zero in this homogeneous boundary value problem (Figure $1)$ :

$$
\left.\lambda \frac{\partial T}{\partial z}\right|_{z=0}=-I\left[T-\left(T_{s}=0\right)\right] \text { or }\left.\frac{\partial T}{\partial z}\right|_{z=0}=-h T \text { with } h=\frac{I}{\lambda}
$$

In Eq. (3), $I\left[W m^{-2} K^{-1}\right]$ is the surface heat transfer coefficient [4]. The coefficient $h\left[m^{-1}\right]$ can be regarded as the coupling coefficient between GST and $T_{S}$ [33]. Theoretically, $h$ can range between 0 (no coupling) and infinite (perfect coupling), representing either full insulation or a Dirichlet-type $\mathrm{BC}$ at the ground surface, respectively. The assumptions of an initially zero 
temperature field as well as $T_{s}=0$ may be relaxed by superposing the associated nonhomogeneous boundary value problem as indicated in $[29,30]$.

Compared with the traditionally prescribed Dirichlet-type BC $\left(T=T_{S}\right)$, Eq. (3) offers a more flexible and realistic representation of near-surface conditions influencing the thermal regime around BHEs. However, it needs to be emphasized that the new parameter $h$ for specifying the Cauchy-type BC serves as surrogate of complex land-atmosphere processes, and so the obtained formulation is still strongly simplifying (e.g. $[36,50])$.

BCs as defined in Eq. (3) are common in numerical heat transport simulations to account for the effect of a rather thin layer that represents the transition between the domain of interest (i.e. the ground) and another medium with known temperature (i.e. the atmosphere) [51]. These known conditions can be transferred to the boundary of a model by assigning a surface heat transfer coefficient, $I=\lambda_{o} / d_{o}$, where $\lambda_{o}$ is the thermal conductivity and $d_{o}$ is the thickness of the transition layer. One shortcoming of this approach is that the thermal inertia of the transition layer is neglected. Still, it is a valuable model for approximating the effect of thin media such as slabs and pavements within large scale models. These thin layers reach steady state thermal conditions relatively early, and thus they do not need to be resolved as layers in the model.

Other near surface conditions such as an unsaturated zone could be accounted for in a similar way if its capacity to store and release heat is negligible. In this case, a sufficiently approximate approach may be to resolve the temperature field in this zone assuming saturated conditions (homogenization), since the thermal diffusivity does not change drastically with water content in porous media. Palmer et al. [40] for instance, based on the experimental work presented in [52], argued that thermal effects of an unsaturated zone are not appreciable for water contents between 20\% and 100\%. More recently, Simms et al. [53] implemented numerical models to evaluate the effect of soil's thermal properties heterogeneity on the performance of horizontal ground heat exchangers. For these very shallow systems, their results support the homogenization approach since the impact of this heterogeneity is minimal compared with uncertainty of soils' mean thermal properties. In general, close to the ground surface, the imposed $\mathrm{BC}$ is likely more dominant than potential effects induced by changes in water content [54].

The problem posed in Eqs. (1)-(3) can be solved analytically by linear superposition in space and time of unitary-instantaneous heat pulses according to the problem-specific Green's function. This is a common procedure for simulating BHEs with simple and complex Dirichlettype BCs (e.g. [29, 30, 55, 56]). In contrast, Cauchy-type BCs have been implemented only for 
simulating heat transfer at the borehole wall but not at the ground surface (e.g. [57]). The new analytical solution builds on a line source model and incorporates Eq. (3) as BC at the ground surface. For the system shown in Figure 1 it reads (see also Appendix A, Eq. A11):

$$
T\left(\boldsymbol{x}, \boldsymbol{x}^{\prime}, t\right)=T_{M F L S}\left(\boldsymbol{x}, \boldsymbol{x}^{\prime}, t\right)+\Delta T_{h}\left(\boldsymbol{x}, \boldsymbol{x}^{\prime}, t\right)
$$

where $T_{M F L S}$ is the temperature calculated by the standard moving finite line source (MFLS) [24], and $\Delta T_{h}$ is the contribution associated with Cauchy-type BC effects given by

$$
\begin{aligned}
\Delta T_{h}\left(\boldsymbol{x}, \boldsymbol{x}^{\prime}, t\right)= & \frac{q}{4 \lambda \pi} \exp \left(\frac{x-x^{\prime}}{2 a} v\right) \int_{\frac{r_{d}^{2}}{4 a t}}^{\infty} \frac{1}{\varphi} \exp (-\varphi \\
& \left.-\left(\frac{r_{d} v}{4 a}\right)^{2} \frac{1}{\varphi}\right)\left\{\operatorname { e x p } ( h z + ( \frac { h r _ { d } } { 2 } ) ^ { 2 } \frac { 1 } { \varphi } ) \left[\operatorname{erfc}\left(\frac{z \sqrt{\varphi}}{r_{d}}+\frac{h r_{d}}{2 \sqrt{\varphi}}\right)\right.\right. \\
& \left.\left.-\exp (h H) \operatorname{erfc}\left(\frac{z+H}{r_{d}} \sqrt{\varphi}+\frac{h r_{d}}{2 \sqrt{\varphi}}\right)\right]\right\} d \varphi
\end{aligned}
$$

where $r_{d}^{2}=\left(x-x^{\prime}\right)^{2}+\left(y-y^{\prime}\right)^{2}$.

Depending on the specific combination of parameters $\left(\boldsymbol{x}, \boldsymbol{x}^{\prime}, t, h, a\right)$, the factor enclosed within the curly brackets in Eq. (5) can generate arithmetic overflow while evaluating the integral. One way around is to include the exponential factors within the complementary error function (erfc) through the following substitution (see also Appendix B, Eq. B3):

$$
\kappa\left(h, \mu, z, r_{d}\right)=\frac{2}{r_{d}} \sqrt{\frac{\varphi}{\pi}} \int_{0}^{\infty} \exp \left[-h \varepsilon-\varphi\left(\frac{z+\mu+\varepsilon}{r_{d}}\right)^{2}\right] d \varepsilon
$$

which leads to reformulate Eq. (5) as

$$
\begin{aligned}
\Delta T_{h \kappa}\left(\boldsymbol{x}, \boldsymbol{x}^{\prime}, t\right) & =\frac{q}{4 \lambda \pi} \exp \left(\frac{x-x^{\prime}}{2 a} v\right) \int_{\frac{r_{d}^{2}}{4 a t}}^{\infty} \frac{1}{\varphi} \exp (-\varphi \\
& \left.-\left(\frac{r_{d} v}{4 a}\right)^{2} \frac{1}{\varphi}\right)\left[\kappa\left(h, 0, z, r_{d}\right)-\kappa\left(h, H, z, r_{d}\right)\right] d \varphi
\end{aligned}
$$

The dimensionless forms of Eq. (4) can be written as

$$
\theta\left(\boldsymbol{X}, \boldsymbol{X}^{\prime}, P e, F o\right)=\theta_{M F L S}\left(\boldsymbol{X}, \boldsymbol{X}^{\prime}, P e, F o\right)+\Delta \theta_{h}\left(\boldsymbol{X}, \boldsymbol{X}^{\prime}, P e, F o, H_{h}\right)
$$


where $\theta$ refers to dimensionless temperature. Alternatively, $\Delta \theta_{h}$ can be expressed as $\Delta \theta_{h \kappa}$ to avoid arithmetic overflow, both formulations are included in the Appendix C (Eqs. C1-C3). In Eq. (8), the indicated dimensionless numbers including the Péclet number $P e$ and the Fourier number $F o$, are defined as follows

$$
\begin{aligned}
& P e=\frac{v H}{a}, \quad F o=\frac{a t}{H^{2}}, \quad\left(\boldsymbol{X}, \boldsymbol{X}^{\prime}\right)=\left(<X, Y, Z>,<X^{\prime}, Y^{\prime}, Z^{\prime}>\right) \\
&=\frac{1}{H}\left(<x, y, z>,<x^{\prime}, y^{\prime}, z^{\prime}>\right), \quad R=\frac{r_{d}}{H}, \quad H_{h}=h H
\end{aligned}
$$

Eqs. (8)-(9) are employed in the next chapter to examine the effect of the two boundary formulations on thermal plume evolution around a BHE (i.e. on $\Delta \theta_{h}$ and $\theta_{M F L S}$ ). Additionally, potential implications of simulation with Cauchy-type BC on optimal BHE sizing are addressed. For this, it is necessary to estimate the borehole wall temperature, which can be expressed through so-called ' $g$-functions' as introduced by Eskilson $[28,44]$ and thoroughly discussed in $[58,59]$. For single BHEs, an accepted metric for this temperature is the mean borehole wall temperature (calculated with the finite line source model) as defined by Zeng et al. [45] and reformulated by Lamarche and Beauchamp [60]. Here, it is proposed an alternative formulation for the mean borehole wall temperature $\overline{T_{M F L S}}$ which is based on the approach presented in [29, 61]:

$$
\overline{T_{M F L S}}=\frac{1}{2 \pi r_{b} H} \int_{0}^{2 \pi} \int_{0}^{H} T_{M F L S} r_{b} d z d \delta=\frac{1}{2 \pi H} \int_{0}^{2 \pi} \int_{0}^{H} T_{M F L S} d z d \delta
$$

where $d \delta$ is the differential angle in cylindrical coordinates and $T_{M F L S}$ is evaluated at the borehole radius. This alternative formulation reads:

$$
\begin{aligned}
\overline{T_{M F L S}}=\frac{q}{8 \lambda \pi} I_{0}\left(\frac{R_{b} P e}{2}\right) \int_{\frac{R_{b}^{2}}{4 F o}}^{\infty} \frac{1}{\varphi} \exp \left[-\varphi-\left(\frac{R_{b} P e}{4}\right)^{2} \frac{1}{\varphi}\right]\left\{4 \operatorname{erf}\left(\frac{\sqrt{\varphi}}{R_{b}}\right)\right. \\
-2 \operatorname{erf}\left(\frac{2 \sqrt{\varphi}}{R_{b}}\right) \\
\left.+\frac{R_{b}}{\sqrt{\pi \varphi}}\left[4 \exp \left(-\frac{\varphi}{R_{b}^{2}}\right)-\exp \left(-\frac{4 \varphi}{R_{b}^{2}}\right)-3\right]\right\} d \varphi
\end{aligned}
$$

where $R_{b}=\frac{r_{b}}{H}$ and $I_{0}$ is the modified Bessel function of first kind and of order zero. Since our interest is to quantify the net effect of Eq. (3) in the mean borehole temperature, Eq. (11) will be compared with an analogous expression derived from $\Delta T_{h}$ (Eq. (5)): 


$$
\begin{aligned}
& \overline{\Delta T_{h}}=\frac{1}{2 \pi r_{b} H} \int_{0}^{2 \pi} \int_{0}^{H} \Delta T_{h} r_{b} d z d \delta=\frac{1}{2 \pi H} \int_{0}^{2 \pi} \int_{0}^{H} \Delta T_{h} d z d \delta \\
& \overline{\Delta T_{h}}=\frac{q}{4 \lambda \pi H_{h}} I_{0}\left(\frac{r_{b}}{2 a} v\right) \int_{\frac{r_{b}^{2}}{4 a t}}^{\infty} \frac{1}{\varphi} \exp \left(-\varphi-\left(\frac{r_{b} v}{4 a}\right)^{2} \frac{1}{\varphi}\right) \psi\left(h, H, r_{b}, \varphi\right) d \varphi \\
& \psi\left(h, H, r_{b}, \varphi\right)=2 \operatorname{erf}\left(\frac{H}{r_{b}} \sqrt{\varphi}\right)-\operatorname{erf}\left(\frac{2 H}{r_{b}} \sqrt{\varphi}\right) \\
& +\exp \left[\left(\frac{h r_{b}}{2}\right)^{2} \frac{1}{\varphi}\right]\left[\exp (h H) \operatorname{erfc}\left(\frac{H}{r_{b}} \sqrt{\varphi}+\frac{h r_{b}}{2 \sqrt{\varphi}}\right)\right. \\
& -\operatorname{erfc}\left(\frac{h r_{b}}{2 \sqrt{\varphi}}\right)-\exp (2 h H) \operatorname{erfc}\left(\frac{2 H}{r_{b}} \sqrt{\varphi}+\frac{h r_{b}}{2 \sqrt{\varphi}}\right) \\
& \left.+\exp (h H) \operatorname{erfc}\left(\frac{H}{r_{b}} \sqrt{\varphi}+\frac{h r_{b}}{2 \sqrt{\varphi}}\right)\right]
\end{aligned}
$$

Alternatively, the function $\psi\left(h, H, r_{b}, \varphi\right)$ in Eq. (14) can be expressed in terms of $\kappa\left(h, \mu, z, r_{d}\right)$ (Eq. (6)) as follows:

$$
\begin{aligned}
\psi_{\kappa}\left(h, H, r_{b}, \varphi\right) & =2 \operatorname{erf}\left(\frac{H}{r_{b}} \sqrt{\varphi}\right)-\operatorname{erf}\left(\frac{2 H}{r_{b}} \sqrt{\varphi}\right)+\kappa\left(h, H, 0, r_{b}\right) \\
& -\kappa\left(h, 0,0, r_{b}\right)-\kappa\left(h, H, H, r_{b}\right)+\kappa\left(h, 0, H, r_{b}\right)
\end{aligned}
$$

In Eqs. (13)-(15), the dimensionless groups shown in Eq. (9) can also be identified. The corresponding dimensionless forms $\overline{\Delta \theta_{h}}, \Psi$ and $\Psi_{\mathrm{K}}$ are listed in Appendix C (Eqs. C4-C6).

In order to compare $\overline{\Delta \theta_{h}}$ with the dimensionless form of Eq. (11) $\left(\overline{\theta_{M F L S}}\right)$, we have $\overline{\theta_{M F L S}}=4 \lambda \pi \overline{T_{M F L S}} / q$.

Finally, a comparison between Dirichlet- and Cauchy-type BCs is carried out by inspecting their effect on the overall ground energy balance during BHE operation. Following the analysis in Rivera at al. [62], the time-dependent power supplied and lost through the top boundary, $p(z=0, t)$, is obtained by integrating the vertical heat fluxes over the entire top surface:

$$
p(z=0, t)=\int_{-\infty}^{\infty} \int_{-\infty}^{\infty} \lambda\left(\frac{\partial T}{\partial z}\right)_{z=0} d x d y=\int_{-\infty}^{\infty} \int_{-\infty}^{\infty} I\left(\Delta T_{h}\right)_{z=0} d x d y
$$


where Eqs. (3) and (4) are merged taking into account that $T_{M F L S}$ is always zero at the top surface, and the assumption $T_{S}=0$ (homogeneous boundary-value problem). The triple integral in Eq. (16) can be simplified as follows [62]:

$$
\begin{aligned}
p(z=0, t)= & \frac{h q H^{2}}{4} \int_{\frac{H^{2}}{4 a t}}^{\infty} \frac{1}{\varphi^{2}} \exp \left[\left(\frac{h H}{2}\right)^{2} \frac{1}{\varphi}\right]\left[\operatorname{erfc}\left(\frac{h H}{2 \sqrt{\varphi}}\right)\right. \\
& \left.-\exp (h H) \operatorname{erfc}\left(\sqrt{\varphi}+\frac{h H}{2 \sqrt{\varphi}}\right)\right] d \varphi
\end{aligned}
$$

or alternatively in terms of the function $\kappa$ (Eq. (6)):

$$
p(z=0, t)=\frac{h q H^{2}}{4} \int_{\frac{H^{2}}{4 a t}}^{\infty} \frac{1}{\varphi^{2}}[\kappa(h, 0,0, H)-\kappa(h, H, 0, H)] d \varphi
$$

The dimensionless form of the total power is then $P\left(F o, H_{h}\right)=p(z=0, t) / q H$

where Fo (appearing in the lower limit of the integral) and $H_{h}$ are defined in Eq. (9).

In the next chapter, the proposed analytical framework is numerically verified and its role elucidated via a sensitivity analysis based on the identified dimensionless groups. For this, focus is set on thermal plumes, mean borehole temperatures ( $g$-functions) and power supplied to the BHE through the top boundary.

\section{Results and discussion}

\subsection{Numerical verification}

A fundamental first step for proving the correctness of the proposed model is its numerical verification. For this, the synthetic setup shown in Figure 1 is simulated using a finite element heat transport model (FEFLOW version 6.2 [51]). Temperature depth profiles (TDPs) are taken at different locations and times, and these are compared to analytical results obtained by Eqs. (4)-(7).

The physical and thermal properties of the porous medium are chosen so that they represent typical values for sandy aquifers [17]: effective porosity $n_{e}=0.25$, bulk thermal conductivity $\lambda$ $=2.4 \mathrm{~W} \mathrm{~m}^{-1} \mathrm{~K}^{-1}$, and volumetric heat capacity of the porous medium $c=2.7 \mathrm{MJ} \mathrm{K}^{-1} \mathrm{~m}^{-3}$. For this verification, the heat rate at the borehole is set to $q=-30 \mathrm{~W} \mathrm{~m}^{-1}$ (heating) with a length $H=50$ 
$\mathrm{m}$. The BHE is simulated in FEFLOW via a linear (vertical) discrete feature element (DFE) that connects multiple nodal sources with equal heat rate $\left(4^{\text {th }}\right.$ kind $\left.\mathrm{BC}\right)$.

FEFLOW allows the implementation of Cauchy-type BC at the ground surface by assigning an input/output heat transfer coefficient to the upper most numerical layer. In this exercise, this coefficient is $I=1 \mathrm{~W} \mathrm{~m}^{-2} \mathrm{~K}^{-1}$ which implies a heat exchange rate $h=I / \lambda=0.4 \mathrm{~m}^{-1}$. Additionally, in the numerical model it is necessary to assign the known temperature of the medium above the ground to the grid nodes constituting the upper most numerical slice (i.e. the ground surface). This temperature, as well as the initial temperature in the domain, are set equal to zero according to mathematical model assumptions (Figure 2). The size of the numerical model is chosen so that lateral and bottom boundary effects are negligible. In the vertical direction, the $100 \mathrm{~m}$ depth synthetic aquifer is discretised in 142 layers with varying thickness. For the first $55 \mathrm{~m}$, the layers are $0.5 \mathrm{~m}$ thick in order to resolve properly the top boundary effects. Then the thickness of the layers is increased to $1 \mathrm{~m}$ for depths between $55 \mathrm{~m}$ and $70 \mathrm{~m}$, and to $2 \mathrm{~m}$ thickness onwards. In the horizontal plane, an area of $200 \mathrm{~m} \times 100 \mathrm{~m}$ is discretised in around 8500 nodes with higher density close to the BHE. This discretization scheme is similar to that applied in [24, 30]. Finally, horizontal groundwater flow is accounted for by lateral fixed head settings and assuming a Darcy flux of $D=1 \times 10^{-7} \mathrm{~m} / \mathrm{s}$.

Figure 3 depicts the temperature profiles calculated with both methodologies, the numerical and the new analytical one. Further testing revealed that the observed minor discrepancies tend to decrease with finer discretization in the numerical model. Nevertheless, the shown accuracy is considered enough to demonstrate the capability of the analytical solution to reproduce the features of the physical model.

For the same study case, Figure 4a-c visualize the components of Eq. (4) after 20 years of operation. The effect of $\Delta T_{h}$ (Figure 4a) is maximal at the junction between the BHE and the ground surface, where extreme thermal gradients occur. Its intensity diminishes quickly with radial distance from this junction. The processes and parameters that control $\Delta T_{h}$ are subsequently analysed through a dimensional analysis.

\subsection{Effect on the thermal plumes of BHEs}

The main feature of the proposed new analytical solution is the possibility to estimate the temperature field in the near-surface domain influenced by the interaction of both the BHE and the surface air temperature (SAT). For more insight, a reference scenario is defined that consists 
of a BHE with $H=100 \mathrm{~m}$ installed in an aquifer with same properties as the study case considered for the numerical verification. The heat extraction rate is kept at $q=-30 \mathrm{~W} \mathrm{~m}^{-1}$ and the groundwater flow is described by $D=4 \times 10^{-7} \mathrm{~m} / \mathrm{s}$ (seepage velocity of around $50 \mathrm{~m} / \mathrm{y}$ ), which corresponds to $P e=70$. This is a rather "rule-of-thumb" value for sandy aquifers [17, $63]$, but it may vary significantly for different cases.

Defining a generally valid benchmark value for the heat flux coupling coefficient at the ground surface is hardy possible. Magnitudes can range between low values such as $h \approx 0.05 \mathrm{~m}^{-1}$ [64, 65] and extremely high ones such $h=23 \mathrm{~m}^{-1}$ [42]. Those reported high values though are likely affected by convective heat input due to infiltrating precipitation through the ground surface [39]. Among others, variables such as wind velocity, season of the year (e.g. snow cover) or specific land use can potentially change the magnitude of the exchange rate. Taking the data from Müller et al. [66] for instance, one can estimate a mean annual heat exchange rate of 0.2 $\mathrm{m}^{-1}$ for the city centre of Oberhausen (Germany), whereas in suburban areas, $h$ ranges between 2 and $8 \mathrm{~m}^{-1}$. In another example, for the city of Nanjing (China), the records from Tang et al. [67] suggest an annual mean value for the urbanized area of $0.3 \mathrm{~m}^{-1}$. In contrast, the conditions in the rural surrounding are described by around $h=0.1 \mathrm{~m}^{-1}$. For comparison purposes, the reference $h$ value is chosen to be $0.5 \mathrm{~m}^{-1}$ following the estimations in Molson et al. and Palmer et al. [39, 40] for the Borden site close to Toronto (Canada) and Güven et al. [37] for the site described in Werner and Kley [64].

In the reference scenario, the parameters and thermal properties are selected so that the dimensionless temperature scale is equivalent to the absolute scale in ${ }^{\circ} \mathrm{C}$. The analysis begins by characterizing $\Delta \theta_{h}$ (Eqs. $\mathrm{C} 1$ or $\left.\mathrm{C} 3\right)$ at the ground surface $(z=0, y=0)$ when the dimensionless groups $P e, F o$ and $H_{h}$ are changed. Figure 5 depicts magnitudes of $\Delta \theta_{h}$ along the positive $x$ (groundwater flow) direction, and the BHE is located at $x=0$.

In Figure 5a, the perturbed length downstream of the BHE is shown at different dimensionless times, $F o$. For the reference scenario, the curves are calculated after $3\left(F o=7 \times 10^{-4}\right)$ and $6(F o$

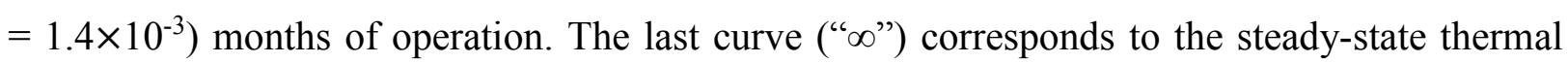
conditions, which prevail after approximately 3.5 years for points with steady-state temperatures higher than $0.1\left({ }^{\circ} \mathrm{C}\right)$ (i.e. distances not longer than $0.40 H$ from the BHE). As expected, Figure 5a indicates that strength and length of the thermal anomaly evolve with time, with maximum values at the BHE location. For the reference scenario, the ground surface is cooled by the BHE operation reaching at most $-2 \mathrm{~K}$ in comparison to the unaffected environment. This anomaly, 
however, becomes negligible for downstream distances longer than the BHE length (here 100 $\mathrm{m})$.

The magnitudes of the Fo numbers and the closeness among the depicted curves imply that steady state conditions are reached relatively fast, especially in the vicinity of the BHE. This is typical for regions near to continuous sources/sinks or to model boundaries. However, the time to reach steady conditions also strongly depends on the magnitude of the $P e$ number.

The role of groundwater flow (GWF) is analysed in more detail in Figure 5b. For this, thermal steady state is assumed and so the maximum temperature changes are inspected. In comparison with the reference case $(P e=70)$, the absence of GWF $(P e=0)$ strengthens the intensity and shortens the perturbed length of the thermal anomaly. The magnitude of those changes indicates that, at the ground surface, the system is relatively insensitive to the advective transport mechanism: while the $P e$ number changes from 0 to 139 implying a relative increase in the seepage velocity to around $100 \mathrm{~m} / \mathrm{y}$, the thermal disturbance at the BHE is only affected by around $0.5 \mathrm{~K}$. Advection also moves the thermal anomaly downwards. Considering a minimum dimensionless temperature change of 0.1 , the scenario without GWF yields higher changes within the first $0.3 H$ (downstream) from the BHE. This distance can increase up to $0.4 H$ for the scenario with $P e=139$. This is again a relatively low change compared with the change in $P e$. Moreover, there exist insignificant differences between the reference case $(P e=70)$ and the scenario with $P e=139$ beyond $x=0.1 H$. This is in general a convenient model feature, since thermal effects from groundwater flow are not expected close the ground surface.

The impact of changing the heat exchange rate $h$ on the temperature at the ground surface is depicted in Figure 5c. The proposed normalized $H_{h}$ values between 10 and 100 are derived by changing $h$ between 0.1 and $1 \mathrm{~m}^{-1}$ while keeping the reference $H=100 \mathrm{~m}$. The curves are obtained assuming again steady-state conditions $(F O=\infty)$ and $P e=70$. In comparison with the $P e$ number, $H_{h}$ has a higher impact on the induced thermal anomaly, as it is indicated by the sensitivities in Figure 5d. By changing $H_{h}$ from 10 to 100 , the dimensionless temperature at the borehole wall varies by around 1.6 ( or $1.6 \mathrm{~K}$ ) with a maximum of 3.0 for $H_{h}=10$. A lower $H_{h}$ implies a higher thermal resistance at the ground surface (or stronger insulating effect). Since heat is extracted from the ground, the recovery of the cooled ground by vertical heat supply thus is slower. With higher $H_{h}$ values, as expected, the effect of $\Delta \theta_{h}$ diminishes and the dimensionless total temperature $\theta$ approaches $\theta_{M F L S}=0$. The thermal affected length downstream is also clearly driven by the value of $H_{h}$. For the threshold of 0.1 as minimum 
dimensionless temperature change, this horizontal distance increases from $X=0.4$ (reference with $\left.H_{h}=50\right)$ to more than one borehole length $(X \geq 1)$ for $H_{h}=10$.

The temporal dynamics of the Cauchy-type $\mathrm{BC}$ contribution in dimensionless form, $\Delta \theta_{h}$, is depicted in Figure 6 for a fixed point $(x=0.1 H, y=0)$ at the ground surface $(z=0)$. This examined point thus is in vicinity of the BHE, e.g. at radial or downstream distance of $10 \mathrm{~m}$ for a $100 \mathrm{~m}$ borehole. Figure 6a corresponds to a scenario without groundwater flow, whereas in Figure $6 \mathrm{~b}$ the previous advective scenario with $P e=70$ (Figure 5a) is kept. Consistent with related studies on BHEs, it is shown that groundwater flow accelerates reaching steady state conditions (e.g. [68, 69]). However, the top boundary condition, specified by $H_{h}$, reveals to be more decisive for the value of the steady-state dimensionless temperature.

The influence of the Cauchy-type boundary condition for $\Delta \theta_{h}$ is not only visible at the ground surface. It is also of interest to characterize its penetration depth and to compare it with the standard moving finite line source (MFLS). Figure 7a illustrates the variability of $\Delta \theta_{h}$ and the ratio $\Delta \theta_{h} / \theta_{M F L S}$ with depth (as temperature depth profiles, TDPs) for the reference scenario at different dimensionless times, $F o$. The responses $\Delta \theta_{h}, \theta_{M F L S}$ are calculated again at location $x$ $=0.1 H$ and $y=0$.

The selected Fo numbers are the same as those shown in Figure 5a (3 and 6 months after operation and steady state thermal conditions) in the reference scenario. The steady-state varies with the horizontal distance from the BHE at which the temperature depth profile is taken. For the chosen profile, and the conditions of the reference case, steady-state is reached after approximately 2 years of operation. By comparing Figure 5a and Figure 7a, it is clear that the damping of the induced thermal anomaly is stronger in the vertical direction than radially at the ground surface. This can be confirmed by contrasting the steady-state curves with the distances measured from the origin of coordinates where $\Delta \theta_{h}$ is maximum (Figure 4). For example, at the ground surface, Figure 5a indicates that $\Delta \theta_{h}$ is negligible for radial distances larger than $H$. For the chosen profile, Figure 7 a reveals that the signal is already negligible for depths of more than $0.15 H$.

The second, right axis of Figure $7 \mathrm{a}$ refers to the ratio $\Delta \theta_{h} / \theta_{M F L S}$, which serves as measure for the discrepancy associated with neglecting Cauchy-type BC effects. This discrepancy is notoriously high for the shallowest part of the domain, where it reaches values above $100 \%$ even for the highest $H_{h}$. This discrepancy though rapidly diminishes with depth. Indeed, at the chosen location of the profile, the discrepancy is more than $10 \%$ only for shallow depths not larger than $0.07 \mathrm{H}$. Here is important to recall that in these results, the dimensionless numbers 
are calculated for a reference borehole length of $100 \mathrm{~m}$. For shorter BHEs, the unitless scales are shifted and the effect of $\Delta \theta_{h}$ could be more relevant (see Figure 8).

The role of groundwater flow (GWF) on the selected profile is assessed in Figure $7 \mathrm{~b}$ for the reference case under steady state conditions $(F o=\infty)$. In contrast to Figure $5 \mathrm{~b}$, here the calculated temperatures are more sensitive to GWF. This is due to the fact that the imposed Cauchy-type boundary condition effect is weaker at deeper locations and therefore with less control on the heat transport processes (advection and conduction). This is exemplified by comparing in Figure $7 \mathrm{~b}$ the total temperature change (black lines) at the ground surface $(z=0)$ and the analogous change at $z=0.15 \mathrm{H}$. The fact that stronger GWF leads to generally lower dimensionless temperature is not surprising, since advection smooths thermal anomalies and generally enhances heat fluxes (e.g. [69]). The trends of the ratio $\Delta \theta_{h} / \theta_{M F L S}$ in Figure $7 \mathrm{~b}$ once again highlight that the different boundary settings have significant influence on the top. This discrepancy is higher than $10 \%$ for the $12 \%$ most superficial part of the domain for the most unfavourable scenario without GWF.

Finally, the influence of $H_{h}$ on $\Delta \theta_{h}$ along the vertical direction is depicted in Figure $7 \mathrm{c}$. The behaviour is analogous to the one shown in Figure 5c (insulation effect), but the damping is so strong that a relatively low $H_{h}=10$ has an impact higher than $0.1(\mathrm{~K})$ for depths not larger than $z=0.1 \mathrm{H}$. Consequently, for the (arbitrary) maximum discrepancy threshold of $10 \%$, the MFLS model would be an acceptable approximation for depths larger than $z=0.09 H$.

The results shown in Figure 7 were obtained assuming a reference length of $H=100 \mathrm{~m}$. Since $\Delta \theta_{h}$ is not linear with respect to $H_{h}$, it is worth quantifying the effect on more shallow BHEs. Figure 8 shows the maximum normalized depth at which $\Delta \theta_{h} / \theta_{M F L S} \geq 0.1$ (or $10 \%$ discrepancy threshold) assuming no groundwater flow (unfavourable case) and a thermal profile taken at $x=$ $0.1 H$ and $y=0$. The discrepancy threshold is given as function of $F o$ and $H_{h}$.

For the considered range of $H_{h}$, Figure 8 indicates that $\theta_{M F L S}$ is an acceptable surrogate to $\theta$ (less than $10 \%$ discrepancy) for normalized depths of $z>0.1 H$ (minor insulation) and $z>0.35 H$ (strong insulation). Taken for instance an energy pile with $H=20 \mathrm{~m}$ such as the one analysed by Loveridge and Powrie [70] and the moderate reference case with $h=0.5 \mathrm{~m}^{-1}$, Figure 8 implies that the MFLS is not acceptable for estimating the temperature at depths ranging between $z=0$ and $z=0.3 H=6 \mathrm{~m}$ at the medium term $\left(F_{O} \geq 0.1\right)$. Of course, the line source model is not the best approach for describing energy piles, where geometry and the thermal inertia of the fill material are important issues. However, since shallower geothermal systems (e.g. energy piles and coils) are even more affected by the thermal conditions at the ground surface than BHEs, 
the proposed Cauchy-type BC might be suited to improve existing analytical models (e.g. [16, $56,68,71,72])$.

\subsection{Effect on the mean borehole wall temperature and total power supply from the ground surface}

Given the generally short noticeable penetration depth of $\Delta \theta_{h}$, its effect on BHE sizing is minor for sufficiently deep installations. This is confirmed in Figure 9, where the ratio $\overline{\Delta \theta_{h}} / \overline{\theta_{M F L S}}$ is calculated after 30 years of operation assuming a borehole radius $r_{b}=0.1 \mathrm{~m}$ and $P e=0$. The ratio is shown as a function of the coefficient $h$ and for different borehole lengths, $H$. A scenario without groundwater flow is chosen as reference, since it maximizes the ratio $\Delta \theta_{h} / \theta_{M F L S}$ yielding the most disadvantageous conditions for $\overline{\theta_{M F L S}}$ as an approximation for $\bar{\theta}$ (Figure $7 \mathrm{~b}$ ). For these conditions and taking a short, only $25 \mathrm{~m}$ long BHE, for instance, Figure 9 indicates that the mean temperature at the borehole can be estimated with the standard MFLS with an associated error of less than $14 \%$ in the worst case. In comparison with Figure 8, the effect of $\Delta \theta_{h}$ is less appreciable here, because at the borehole wall it is overprint by the line source heat extraction rate even for rather shallow BHEs.

The effect of the BCs given by Eq. (3) on the total power supplied from the ground surface is assessed in Figure 10. During BHE operation, the temperature distribution in the ground is determined by a ground energy balance that considers the harnessed energy $(q)$, the thermal exhaustion of the subsurface (also the contribution from groundwater flow), the local geothermal flux and the vertical heat fluxes at the ground surface [17]. The latter become more relevant in the long term, but their magnitude strongly depends on how the boundary condition is simulated. As Figure 10 indicates, for the heating reference scenario $(q<0)$, the assumption of Dirichlet-type BC $\left(H_{h} \rightarrow \infty\right)$ implies the maximum power supply through the top boundary. The figure also shows the convergence between Cauchy- and Dirichlet-type BCs when increasing $H_{h}$. As illustration, taking $F o=0.15$ and the Dirichlet-type BC, around $40 \%$ of the energy extracted by the BHE is provided through the ground surface. This share though diminishes to around $26 \%$ for $H_{h}=5$.

Rivera et al. [62] assumed a Dirichlet-type BC at the ground surface for carrying out a ground energy balance around a BHE represented as a finite line source. It was shown that thermal reservoir depletion is the main source of power during BHE operation. Its share however, decreased with time as the contribution from the top boundary becomes relevant (Figure 10). Implementing a more realistic $\mathrm{BC}$ as proposed here, would imply a smaller power input through 
the top boundary, which in turn leads to a longer and more stressed demand of energy from the reservoir.

\section{Conclusions}

For having more flexibility at the top boundary, for the first time, a line source based formulation for borehole heat exchanger (BHE) simulation is presented that implements a Cauchy-type boundary condition. An elementary feature of this type of boundary condition is that a linear heat transfer across the ground surface is specified. This offers advantages in comparison to the more common fixed temperature assumption for the ground surface, because the degree of coupling between surface air temperature and ground surface temperature can be simulated. The coupling coefficient determines whether the surface-near thermal regime in the ground is isolated or fully synchronized with the atmosphere. This is a crucial point, since operation of BHEs is hardly ever balanced, and thermal anomalies growing in the ground are partially replenished by heat flux through the top boundary. Consequently, accurate and casespecific handling of this boundary is desirable.

The presented analytical model is successfully verified with a numerical model for simulating the transient three-dimensional thermal regime that evolves around a BHE employed for heat extraction. By comparison with the existing standard MFLS, it is revealed that in shallow depths the discrepancies between simulated temperatures are considerable. For the verification case, for example it is expected 2-4 K colder conditions for the Cauchy-type close to the ground surface. These differences are generally more pronounced when horizontal groundwater flow is absent. Still, the noticeable penetration depth of the top boundary condition is limited and especially for long BHEs the error introduced by a fixed temperature condition at the top boundary may be acceptable. For instance, given the BHE of $100 \mathrm{~m}$ in the study case, only for the upper $9 \mathrm{~m}$ discrepancies exceed $10 \%$ for a measurement point at a lateral distance of $10 \mathrm{~m}$. The mean borehole wall temperature is also inspected as commonly modelled by g-functions. It is shown that the difference from different boundary conditions are generally smaller than $5 \%$ for sufficiently long BHEs $(>50 \mathrm{~m})$. The role of appropriate boundary specification, however, is crucial for shorter installations such as energy piles or coils. Since these are similarly modelled by superimposed Green's functions, this study reveals that to top boundary has to be critically dealt with.

Even if the ground surface conditions may not be relevant when simulating the integral performance of BHEs, regulatory frameworks for shallow geothermal systems generally try to mitigate induced thermal anomalies. The correct specification of the top boundary thus may be a 
major concern when consequences on shallower infrastructure such as drinking water supply networks are regulated. Furthermore, guidelines for proper long-term BHE operation emphasize the need for sustainable ground use, and here the energy balance in the ground plays a major role. Since the ground heat flux is an elementary component in this balance, near surface effects are sensible. In the dimensionless analysis it is revealed that the contribution from the top may be reduced by up to $35 \%$. Upcoming work will be dedicated to extend the current solution including the natural geothermal gradient (initial conditions) and non-uniformity in the atmospheric/indoor temperatures. 


\section{Appendix A:}

For an unitary and instantaneous energy pulse released at the point $\boldsymbol{x}$ ' and time $\tau$ in a semiinfinite and homogeneous domain with specified coupling coefficient $h$ through the top boundary, Carslaw and Jeager [4] defined the following Green's function:

$$
\begin{aligned}
G\left(\boldsymbol{x}^{\prime}, \boldsymbol{x}, t-\tau\right) & =\frac{1}{8[a \pi(t-\tau)]^{\frac{3}{2}}}\left\{\exp \left[-\frac{\left(z-z^{\prime}\right)^{2}}{4 a(t-\tau)}\right]\right. \\
& \left.+\exp \left[-\frac{\left(z+z^{\prime}\right)^{2}}{4 a(t-\tau)}\right]\right\} \exp \left[-\frac{r_{d}^{2}}{4 a(t-\tau)}\right] \\
& -\frac{h}{4 a \pi(t-\tau)} \operatorname{erfc}\left[\frac{z+z^{\prime}}{2 \sqrt{a(t-\tau)}}+h \sqrt{a(t-\tau)}\right] \exp \left[h\left(z+z^{\prime}\right)\right. \\
& \left.+a h^{2}(t-\tau)-\frac{r_{d}^{2}}{4 a(t-\tau)}\right]
\end{aligned}
$$

Under the conditions described above, Eq. A1 yields the temperature at any point $\boldsymbol{x}$ and time $t$. For a continuous line source with unitary strength and length $H$, the superposition in space and time reads:

$$
\int_{\tau} \int_{z^{\prime}} G\left(\boldsymbol{x}^{\prime}, \boldsymbol{x}, t-\tau\right) d z^{\prime} d \tau=\int_{\tau} \int_{z^{\prime}}\left[G_{1}\left(\boldsymbol{x}^{\prime}, \boldsymbol{x}, t-\tau\right)+G_{2}\left(\boldsymbol{x}^{\prime}, \boldsymbol{x}, t-\tau\right)\right] d z^{\prime} d \tau
$$

The two integrals on the right hand side of Eq. A2 are defined as follows:

$$
\begin{aligned}
& \int_{\tau} \int_{z^{\prime}} G_{1}\left(x^{\prime}, x, t-\tau\right) d z^{\prime} d \tau \\
& =\frac{1}{8[a \pi]^{3 / 2}} \int_{0}^{t} \frac{1}{(t-\tau)^{3 / 2}} \exp \left[-\frac{r_{d}^{2}}{4 a(t-\tau)}\right] \int_{0}^{H}\left\{\exp \left[-\frac{\left(z-z^{\prime}\right)^{2}}{4 a(t-\tau)}\right]\right. \\
& \left.+\exp \left[-\frac{\left(z+z^{\prime}\right)^{2}}{4 a(t-\tau)}\right]\right\} d z^{\prime} d \tau
\end{aligned}
$$




$$
\begin{aligned}
\int_{\tau} \int_{z^{\prime}} G_{2}\left(\boldsymbol{x}^{\prime}, \boldsymbol{x}, t-\tau\right) d z^{\prime} d \tau \\
=-\frac{h}{4 a \pi} \int_{0}^{t} \frac{1}{t-\tau} \int_{0}^{H} \operatorname{erfc}\left[\frac{z+z^{\prime}}{2 \sqrt{a(t-\tau)}}+h \sqrt{a(t-\tau)}\right] \exp \left[h\left(z+z^{\prime}\right)\right. \\
\left.+a h^{2}(t-\tau)-\frac{r_{d}^{2}}{4 a(t-\tau)}\right] d z^{\prime} d \tau
\end{aligned}
$$

In Eq. A3, the inner integral over $z$ ' can be evaluated yielding:

$$
\begin{aligned}
\int_{\tau} \int_{z^{\prime}} G_{1}\left(\boldsymbol{x}^{\prime}, \boldsymbol{x}, t\right. & -\tau) d z^{\prime} d \tau \\
& =\frac{1}{8 a \pi} \int_{0}^{t} \frac{1}{t-\tau} \exp \left[-\frac{r_{d}^{2}}{4 a(t-\tau)}\right]\left\{\operatorname{erf}\left[\frac{z+H}{\sqrt{4 a(t-\tau)}}\right]\right. \\
& \left.-\operatorname{erf}\left[\frac{z-H}{\sqrt{4 a(t-\tau)}}\right]\right\} d \tau
\end{aligned}
$$

Regarding Eq. A4, this can be reorganized as follows:

$$
\begin{aligned}
& \int_{\tau} \int_{z^{\prime}} G_{2}\left(\boldsymbol{x}^{\prime}, \boldsymbol{x}, t-\tau\right) d z^{\prime} d \tau \\
& =-\frac{h}{4 a \pi} \int_{0}^{t} \frac{1}{t-\tau} \exp \left[a h^{2}(t-\tau)-\frac{r_{d}^{2}}{4 a(t-\tau)}\right] \int_{0}^{H} \operatorname{erfc}\left[\frac{z+z^{\prime}}{2 \sqrt{a(t-\tau)}} \quad\right. \text { A6 } \\
& +h \sqrt{a(t-\tau)}] \exp \left[h\left(z+z^{\prime}\right)\right] d z^{\prime} d \tau
\end{aligned}
$$

By defining the variables $\beta=\sqrt{4 a(t-\tau)}$ and $\sigma=\frac{z+z^{\prime}}{2 \beta}+h \beta$, the integral over $z^{\prime}$ in Eq. A6 is expressed as: 


$$
\begin{gathered}
\int_{0}^{H} \operatorname{erfc}\left[\frac{z+z^{\prime}}{2 \sqrt{a(t-\tau)}}+h \sqrt{a(t-\tau)}\right] \exp \left[h\left(z+z^{\prime}\right)\right] d z^{\prime} \\
=2 \beta \cdot \exp \left(-2 h^{2} \beta^{2}\right) \int_{\frac{z}{2 \beta}+h \beta}^{\frac{z+H}{2 \beta}+h \beta} \operatorname{erfc}(\sigma) \cdot \exp (2 \beta \sigma h) d \sigma
\end{gathered}
$$

The right hand side in A7 can be analytically evaluated yielding:

$$
\begin{gathered}
2 \beta \cdot \exp \left(-2 h^{2} \beta^{2}\right) \int_{\frac{z}{2 \beta}+h \beta}^{\frac{z+H}{2 \beta}+h \beta} \operatorname{erfc}(\sigma) \cdot \exp (2 \beta \sigma h) d \sigma \\
=\frac{\exp \left(-h^{2} \beta^{2}\right)}{h}\left\{\operatorname { e x p } ( h z + h ^ { 2 } \beta ^ { 2 } ) \left[\exp (h H) \operatorname{erfc}\left(\frac{z+H}{2 \beta}+h \beta\right)\right.\right. \\
\left.\left.-\operatorname{erfc}\left(\frac{z}{2 \beta}+h \beta\right)\right]+\operatorname{erf}\left(\frac{z+H}{2 \beta}\right)-\operatorname{erf}\left(\frac{z}{2 \beta}\right)\right\}
\end{gathered}
$$

Substituting A8 in A6 we get:

$$
\begin{aligned}
\int_{\tau} \int_{z^{\prime}} G_{2}\left(\boldsymbol{x}^{\prime}, \boldsymbol{x}, t\right. & -\tau) d z^{\prime} d \tau \\
& =-\frac{1}{4 a \pi} \int_{0}^{t} \frac{1}{t-\tau} \exp \left[-\frac{r_{d}^{2}}{4 a(t-\tau)}\right]\{\exp (h z \\
& \left.+h^{2} \beta^{2}\right)\left[\exp (h H) \operatorname{erfc}\left(\frac{z+H}{2 \beta}+h \beta\right)-\operatorname{erfc}\left(\frac{z}{2 \beta}+h \beta\right)\right] \\
& \left.+\operatorname{erf}\left(\frac{z+H}{2 \beta}\right)-\operatorname{erf}\left(\frac{z}{2 \beta}\right)\right\} d \tau
\end{aligned}
$$

As common for line source models, groundwater flow is accounted via the moving source method, i.e. by changing $x$ with $x-v(t-\tau)$. Furthermore, with the change of variable $\varphi=$ $\frac{r_{d}^{2}}{4 a(t-\tau)}$, Eq. A9 becomes: 


$$
\begin{aligned}
\int_{\tau} \int_{z^{\prime}} G_{2}\left(\boldsymbol{x}^{\prime}, \boldsymbol{x}, t\right. & -\tau) d z^{\prime} d \tau \\
& =-\frac{1}{4 a \pi} \exp \left[-\frac{\left(x-x^{\prime}\right)}{2 a} v\right] \int_{\frac{r_{d}^{2}}{4 a t}}^{\infty} \frac{1}{\varphi} \exp \left[-\varphi-\frac{1}{\varphi}\left(\frac{r_{d}^{2} \cdot v}{4 a}\right)^{2}\right]\{\exp [h z \\
& \left.+\frac{1}{\varphi}\left(\frac{h r_{d}^{2}}{2}\right)^{2}\right]\left[\exp (h H) \operatorname{erfc}\left(\frac{z+H}{r_{d}} \sqrt{\varphi}+\frac{h r_{d}}{2 \sqrt{\varphi}}\right)\right. \\
& \left.\left.-\operatorname{erfc}\left(\frac{z}{r_{d}} \sqrt{\varphi}+\frac{h r_{d}}{2 \sqrt{\varphi}}\right)\right]+\operatorname{erf}\left(\frac{z+H}{r_{d}} \sqrt{\varphi}\right)-\operatorname{erf}\left(\frac{z}{r_{d}} \sqrt{\varphi}\right)\right\} d \tau
\end{aligned}
$$

Taking Eq. A5 and Eq. A10 for a given heat injection/extraction rate $q$, Eq. A2 becomes:

$$
\begin{aligned}
\int_{\tau} \int_{z^{\prime}} G\left(\boldsymbol{x}^{\prime}, \boldsymbol{x}, t\right. & -\tau) d z^{\prime} d \tau \\
& =\frac{q}{4 \lambda \pi} \exp \left[-\frac{\left(x-x^{\prime}\right)}{2 a} v\right] \int_{\frac{r_{d}^{2}}{4 a t}}^{\infty} \frac{1}{\varphi} \exp [-\varphi \\
& \left.-\frac{1}{\varphi}\left(\frac{r_{d}^{2} \cdot v}{4 a}\right)^{2}\right]\left\{\operatorname{erf}\left(\frac{z}{r_{d}} \sqrt{\varphi}\right)-\frac{1}{2} \operatorname{erf}\left(\frac{z-H}{r_{d}} \sqrt{\varphi}\right)\right. \\
& -\frac{1}{2} \operatorname{erf}\left(\frac{z+H}{r_{d}} \sqrt{\varphi}\right) \\
& -\exp \left[h z+\frac{1}{\varphi}\left(\frac{h r_{d}^{2}}{2}\right)^{2}\right]\left[\exp (h H) \operatorname{erfc}\left(\frac{z+H}{r_{d}} \sqrt{\varphi}+\frac{h r_{d}}{2 \sqrt{\varphi}}\right)\right. \\
& \left.\left.-\operatorname{erfc}\left(\frac{z}{r_{d}} \sqrt{\varphi}+\frac{h r_{d}}{2 \sqrt{\varphi}}\right)\right]\right\} d \tau
\end{aligned}
$$

According to [62], the standard MFLS can be rewritten as:

$$
\begin{aligned}
T_{M F L S}\left(\boldsymbol{x}, \boldsymbol{x}^{\prime}, t\right) & =\frac{q}{8 \lambda \pi} \exp \left(\frac{x-x^{\prime}}{2 a} v\right) \int_{\frac{r_{d}^{2}}{4 a t}}^{\infty} \frac{1}{\varphi} \exp \left[-\varphi-\left(\frac{r_{d} v}{4 a}\right)^{2} \frac{1}{\varphi}\right]\left\{2 \operatorname{erf}\left(\frac{z}{r_{d}} \sqrt{\varphi}\right)\right. \\
& \left.-\operatorname{erf}\left(\frac{z-H}{r_{d}} \sqrt{\varphi}\right)-\operatorname{erf}\left(\frac{z+H}{r_{d}} \sqrt{\varphi}\right)\right\} d \varphi
\end{aligned}
$$


Therefore, Eq. A11 can be split as indicated in Eqs. (4)-(5).

\section{Appendix B:}

Defining a function $\kappa\left(h, \mu, z, r_{d}\right)$ as follows:

$$
\kappa\left(h, \mu, z, r_{d}\right)=\exp \left(h z+h \mu+\left(\frac{h r_{d}}{2}\right)^{2} \frac{1}{\varphi}\right)\left[\operatorname{erfc}\left(\frac{z+\mu}{r_{d}} \sqrt{\varphi}+\frac{h r_{d}}{2 \sqrt{\varphi}}\right)\right]
$$

where the definition of the complementary error function leads to:

$$
\kappa\left(h, \mu, z, r_{d}\right)=\exp \left(h z+h \mu+\left(\frac{h r_{d}}{2}\right)^{2} \frac{1}{\varphi}\right) \cdot \frac{2}{\sqrt{\pi}} \int_{\rho}^{\infty} \exp \left(-\delta^{2}\right) d \delta
$$

with $\rho=\frac{z+\mu}{r_{d}} \sqrt{\varphi}+\frac{h r_{d}}{2 \sqrt{\varphi}}$. By substituting $\delta=\frac{z+\mu+\epsilon}{r_{d}} \sqrt{\varphi}+\frac{h r_{d}}{2 \sqrt{\varphi}}$ we have:

$$
\begin{gathered}
\kappa\left(h, \mu, z, r_{d}\right)=\frac{2}{\sqrt{\pi}} \int_{0}^{\infty} \exp \left[-\left(\frac{z+\mu+\epsilon}{r_{d}} \sqrt{\varphi}\right)^{2}-h \epsilon\right] \frac{\sqrt{\varphi}}{r_{d}} d \epsilon \\
= \\
\frac{2}{r_{d}} \sqrt{\frac{\varphi}{\pi}} \int_{0}^{\infty} \exp \left[-\left(\frac{z+\mu+\epsilon}{r_{d}} \sqrt{\varphi}\right)^{2}-h \epsilon\right] d \epsilon
\end{gathered}
$$

Using Eq. B3 for instance, the curly bracket in Eq. (5) can be rewritten as:

$$
\begin{gathered}
\exp \left(h z+\left(\frac{h r_{d}}{2}\right)^{2} \frac{1}{\varphi}\right)\left[\operatorname{erfc}\left(\frac{z \sqrt{\varphi}}{r_{h}}+\frac{h r_{d}}{2 \sqrt{\varphi}}\right)-\exp (h H) \operatorname{erfc}\left(\frac{z+H}{r_{d}} \sqrt{\varphi}+\frac{h r_{d}}{2 \sqrt{\varphi}}\right)\right] \\
=\kappa\left(h, 0, z, r_{d}\right)-\kappa\left(h, H, z, r_{d}\right)
\end{gathered}
$$

\section{Acknowledgements}

This work was supported by the Swiss National Science Foundation (SNSF) under grant number 200021L 144288, and the German Research Foundation (DFG), under grant number BL 1015/4-1. 


\section{References}

1. Zheng, K., et al. Speeding up Industrialized Development of Geothermal Resources in China-Country Update Report 2010-2014. in Proceeding, World Geothermal Congress, 2015,. 2015. Melbourne, Australia.

2. Bayer, P., et al., Greenhouse gas emission savings of ground source heat pump systems in Europe: A review. Renewable and Sustainable Energy Reviews, 2012. 16(2): p. 1256-1267.

3. Lund, J.W. and T.L. Boyd, Direct utilization of geothermal energy 2015: worldwide review, in Proceedings World Geothermal Congress 2015 2015: Melbourne, Australia p. 1-31.

4. Carslaw, H. and J. Jaeger, Conduction of Heat in Solids. second ed. 1959, New York: Oxford University Press.

5. Ingersoll, L., O. Zobel, and A. Ingersoll, Heat Conduction with Engineering, Geological and Other Applications. 1954, New York Mcgraw-Hill.

6. $\quad$ Kobayashi, H., et al., Underground heat flow patterns for dense neighborhoods with heat pumps. International Journal of Heat and Mass Transfer, 2013. 62: p. 632-637.

7. Bayer, P., M. de Paly, and M. Beck, Strategic optimization of borehole heat exchanger field for seasonal geothermal heating and cooling. Applied Energy, 2014. 136: p. 445-453.

8. Hahnlein, S., et al., Sustainability and policy for the thermal use of shallow geothermal energy. Energy Policy, 2013. 59: p. 914-925.

9. Zanchini, E., S. Lazzari, and A. Priarone, Long-term performance of large borehole heat exchanger fields with unbalanced seasonal loads and groundwater flow. Energy, 2012. 38(1): p. 66-77.

10. Brown, E., et al., What are the key issues regarding the role of geothermal energy in meeting energy needs in the global south? 2012.

11. Marcotte, D. and P. Pasquier, Fast fluid and ground temperature computation for geothermal ground-loop heat exchanger systems. Geothermics, 2008. 37(6): p. 651-665.

12. Li, M. and A.C. Lai, Analytical model for short-time responses of ground heat exchangers with $U$ shaped tubes: Model development and validation. Applied Energy, 2013. 104: p. 510-516.

13. Erol, S., M.A. Hashemi, and B. François, Analytical solution of discontinuous heat extraction for sustainability and recovery aspects of borehole heat exchangers. International Journal of Thermal Sciences, 2015. 88: p. 47-58.

14. Lamarche, L. and B. Beauchamp, New solutions for the short-time analysis of geothermal vertical boreholes. International Journal of Heat and Mass Transfer, 2007. 50(7-8): p. 1408-1419.

15. Li, M. and A.C. Lai, Heat-source solutions to heat conduction in anisotropic media with application to pile and borehole ground heat exchangers. Applied Energy, 2012. 96: p. 451-458.

16. Li, M. and A.C. Lai, New temperature response functions ( $G$ functions) for pile and borehole ground heat exchangers based on composite-medium line-source theory. Energy, 2012. 38(1): p. 255-263.

17. Stauffer, F., et al., Thermal use of shallow groundwater. 2013: CRC Press.

18. Wang, H., et al., Thermal performance of borehole heat exchanger under groundwater flow: a case study from Baoding. Energy and Buildings, 2009. 41(12): p. 1368-1373.

19. Hecht-Méndez, J., et al., Optimization of energy extraction for vertical closed-loop geothermal systems considering groundwater flow. Energy Conversion and Management, 2013. 66: p. 1-10.

20. Sutton, M., D. Nutter, and R. Couvillion, A ground resistance for vertical bore heat exchangers with groundwater flow. Journal of Energy Resources Technology-Transactions of The ASME, 2003. 125(3): p. 183-189.

21. Diao, N., Q. Li, and Z. Fang, Heat transfer in ground heat exchangers with groundwater advection. International Journal of Thermal Sciences, 2004. 43(12): p. 1203-1211.

22. Zubair, S. and M.A. Chaudhry, A unified approach to closed-form solutions of moving heat-source problems. Heat and mass transfer, 1998. 33(5-6): p. 415-424.

23. Molina-Giraldo, N., P. Bayer, and P. Blum, Evaluating the influence of thermal dispersion on temperature plumes from geothermal systems using analytical solutions. International Journal of Thermal Sciences, 2011. 50(7): p. 1223-1231.

24. Molina-Giraldo, N., et al., A moving finite line source model to simulate borehole heat exchangers with groundwater advection. International Journal of Thermal Sciences, 2011. 50(12): p. 2506-2513.

25. Marcotte, D., et al., The importance of axial effects for borehole design of geothermal heat-pump systems. Renewable Energy, 2010. 35(4): p. 763-770. 
26. Beck, M., et al., Geometric arrangement and operation mode adjustment in low-enthalpy geothermal borehole fields for heating. Energy, 2013. 49: p. 434-443.

27. Cimmino, M. and M. Bernier, A semi-analytical method to generate g-functions for geothermal bore fields. International Journal of Heat and Mass Transfer, 2014. 70: p. 641-650.

28. Claesson, J. and P. Eskilson, Conductive heat extraction to a deep borehole: Thermal analyses and dimensioning rules. Energy, 1988. 13(6): p. 509-527.

29. Bandos, T., et al., Finite line-source model for borehole heat exchangers: effect of vertical temperature variations. Geothermics, 2009. 38(2): p. 263-270.

30. Rivera, J.A., P. Blum, and P. Bayer, Analytical simulation of groundwater flow and land surface effects on thermal plumes of borehole heat exchangers. Applied Energy, 2015. 146(0): p. 421-433.

31. Goodrich, L., The influence of snow cover on the ground thermal regime. Canadian geotechnical journal, 1982. 19(4): p. 421-432.

32. Zhang, T., Influence of the seasonal snow cover on the ground thermal regime: An overview. Reviews of Geophysics, 2005. 43(4).

33. Stieglitz, M. and J.E. Smerdon, Characterizing land-atmosphere coupling and the implications for subsurface thermodynamics. Journal of climate, 2007. 20(1): p. 21-37.

34. Özış1k, M.N., Boundary value problems of heat conduction. 1989: Courier Corporation.

35. Pollack, H.N., J.E. Smerdon, and P.E. Van Keken, Variable seasonal coupling between air and ground temperatures: A simple representation in terms of subsurface thermal diffusivity. Geophysical Research Letters, 2005. 32(15).

36. Herb, W.R., et al., Ground surface temperature simulation for different land covers. Journal of Hydrology, 2008. 356(3): p. 327-343.

37. Güven, O., J. Melville, and F. Molz, An analysis of the effect of surface heat exchange on the thermal behavior of an idealized aquifer thermal energy storage system. Water Resources Research, 1983. 19(3): p. 860-864.

38. Kumar, R.R., D. Ramana, and R. Singh, Modelling near subsurface temperature with mixed type boundary condition for transient air temperature and vertical groundwater flow. Journal of earth system science, 2012. 121(5): p. 1177-1184.

39. Molson, J.W., E.O. Frind, and C.D. Palmer, Thermal energy storage in an unconfined aquifer: 2. Model development, validation, and application. Water Resources Research, 1992. 28(10): p. 28572867.

40. Palmer, C.D., et al., Thermal energy storage in an unconfined aquifer: 1. Field injection experiment. Water Resources Research, 1992. 28(10): p. 2845-2856.

41. Andrews, C.B., The Impact of the Use of Heat Pumps on Ground-Water Temperatures. Groundwater, 1978. 16(6): p. 437-443.

42. Eugster, W., Erdwärmesonden-Funktionsweise und Wechselwirkung mit dem geologischen Untergrund. 1991, Diss. Naturwiss. ETH Zürich, Nr. 9524, 1991.

43. Zarrella, A. and M. De Carli, Heat transfer analysis of short helical borehole heat exchangers. Applied Energy, 2013. 102: p. 1477-1491.

44. Eskilson, P., Thermal analysis of heat extraction boreholes. Ph.D. Thesis, University of Lund, Lund, Sweden, 1987.

45. Zeng, H., N. Diao, and Z. Fang, A finite line-source model for boreholes in geothermal heat exchangers. Heat Transfer-Asian Research, 2002. 31(7): p. 558-567.

46. Li, M. and A.C.K. Lai, Review of analytical models for heat transfer by vertical ground heat exchangers (GHEs): A perspective of time and space scales. Applied Energy, 2015. 151(0): p. 178191.

47. Anderson, M.P., Heat as a ground water tracer. Groundwater, 2005. 43(6): p. 951-968.

48. García-Gil, A., et al., The thermal consequences of river-level variations in an urban groundwater body highly affected by groundwater heat pumps. Science of The Total Environment, 2014. 485486(0): p. 575-587.

49. Fujii, H., et al., Development of suitability maps for ground-coupled heat pump systems using groundwater and heat transport models. Geothermics, 2007. 36(5): p. 459-472.

50. Deardorff, J., Efficient prediction of ground surface temperature and moisture, with inclusion of a layer of vegetation. Journal of Geophysical Research: Oceans (1978-2012), 1978. 83(C4): p. 18891903.

51. Diersch, H.-J., FEFLOW: Finite element modeling of flow, mass and heat transport in porous and fractured media. 2013: Springer Science \& Business Media. 
52. Moench, A. and D. Evans, Thermal conductivity and diffusivity of soil using a cylindrical heat source. Soil Science Society of America journal, 1970. 34(3): p. 377-381.

53. Simms, R.B., S.R. Haslam, and J.R. Craig, Impact of soil heterogeneity on the functioning of horizontal ground heat exchangers. Geothermics, 2014. 50(0): p. 35-43.

54. Molina-Giraldo, N., et al., Propagation of seasonal temperature signals into an aquifer upon bank infiltration. Groundwater, 2011. 49(4): p. 491-502.

55. Diao, N., Q. Li, and Z. Fang, An analytical solution of the temperature response in geothermal heat exchangers with groundwater advection [J]. Journal of Shandong Institute of Architecture and Engineering, 2003. 3: p. 000.

56. Li, M., et al., Full-scale temperature response function (G-function) for heat transfer by borehole ground heat exchangers (GHEs) from sub-hour to decades. Applied Energy, 2014. 136: p. 197-205.

57. Li, M. and A.C. Lai, Analytical solution to heat conduction in finite hollow composite cylinders with a general boundary condition. International Journal of Heat and Mass Transfer, 2013. 60: p. 549556.

58. Cimmino, M., M. Bernier, and F. Adams, A contribution towards the determination of $g$-functions using the finite line source. Applied Thermal Engineering, 2013. 51(1): p. 401-412.

59. Lazzarotto, A., A network-based methodology for the simulation of borehole heat storage systems. Renewable energy, 2014. 62: p. 265-275.

60. Lamarche, L. and B. Beauchamp, A new contribution to the finite line-source model for geothermal boreholes. Energy and Buildings, 2007. 39(2): p. 188-198.

61. Claesson, J. and S. Javed, An analytical method to calculate borehole fluid temperatures for timescales from minutes to decades. ASHRAE Transactions., 2011. 117(2): p. 279-288.

62. Rivera, J.A., P. Blum, and P. Bayer, Ground energy balance for borehole heat exchangers: Vertical fluxes, groundwater and storage. Renewable Energy, 2015. 83(0): p. 1341-1351.

63. Tye-Gingras, M. and L. Gosselin, Generic ground response functions for ground exchangers in the presence of groundwater flow. Renewable Energy, 2014. 72: p. 354-366.

64. Werner, D. and W. Kley, Problems of heat storage in aquifers. Journal of Hydrology, 1977. 34(1): p. $35-43$.

65. Epting, J., F. Händel, and P. Huggenberger, Thermal management of an unconsolidated shallow urban groundwater body. Hydrology and Earth System Sciences, 2013. 17(5): p. 1851-1869.

66. Müller, N., W. Kuttler, and A.-B. Barlag, Analysis of the subsurface urban heat island in Oberhausen, Germany. Climate research, 2014. 58(3): p. 247-256.

67. Tang, C.-S., et al., Urbanization effect on soil temperature in Nanjing, China. Energy and Buildings, 2011. 43(11): p. 3090-3098.

68. Zhang, W., et al., The analysis on solid cylindrical heat source model of foundation pile ground heat exchangers with groundwater flow. Energy, 2013. 55: p. 417-425.

69. Chiasson, A.D., S.J. Rees, and J.D. Spitler, A Preliminary Assessment Of The Effects Of GroundWater Flow On Closed-Loop Ground-Source Heat Pump Systems. ASHRAE Transactions, 2000. 106(1): p. 380-393.

70. Loveridge, F. and W. Powrie, Temperature response functions (G-functions) for single pile heat exchangers. Energy, 2013. 57: p. 554-564.

71. $\mathrm{Hu}, \mathrm{P}$., et al., A composite cylindrical model and its application in analysis of thermal response and performance for energy pile. Energy and Buildings, 2014. 84: p. 324-332.

72. Bandos, T., et al., Finite cylinder-source model for energy pile heat exchangers: Effects of thermal storage and vertical temperature variations. Energy, 2014. 78: p. 639-648. 


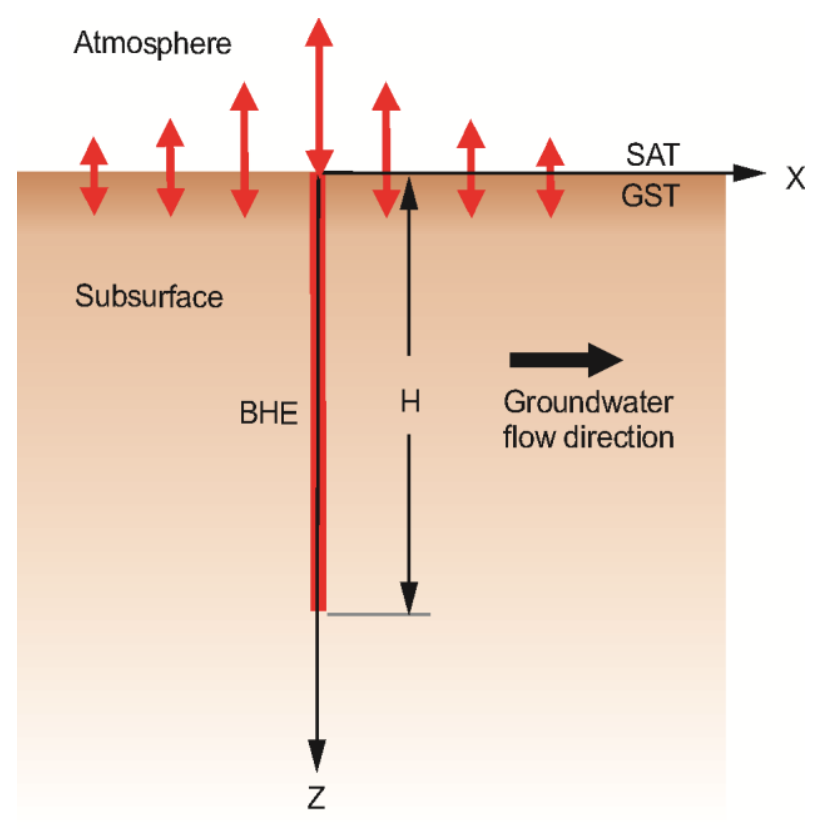

Figure 1: BHE represented as a line source in a semi-infinite space with decoupled surface air temperature (SAT) and ground surface temperature (GST). The red arrows sketch heat fluxes at the ground surface. Downward fluxes occur when SAT>GST.

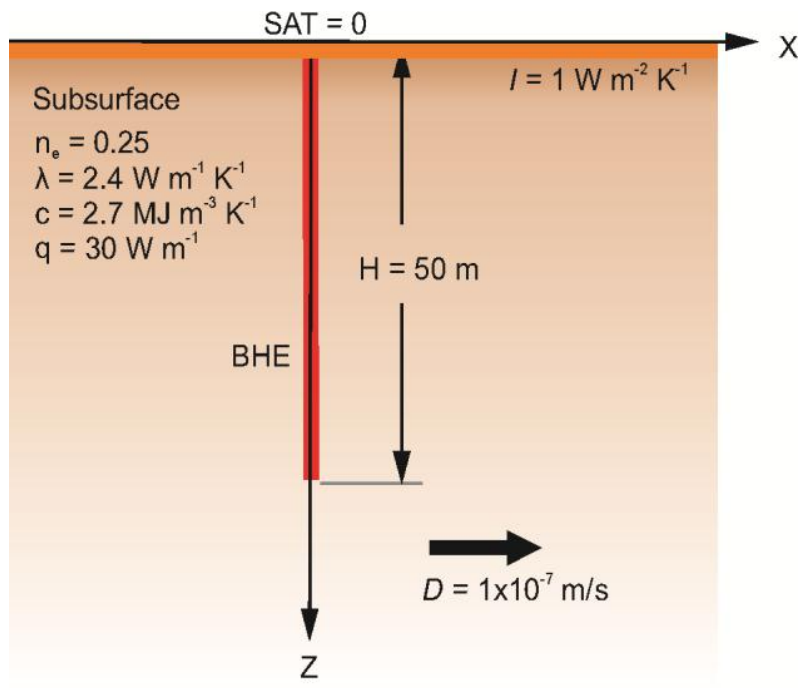

Figure 2: Parameters for the numerical model in FEFLOW. $D$ is Darcy flux and the heat extraction rate is $q$. The initial temperature in the whole domain is zero. 

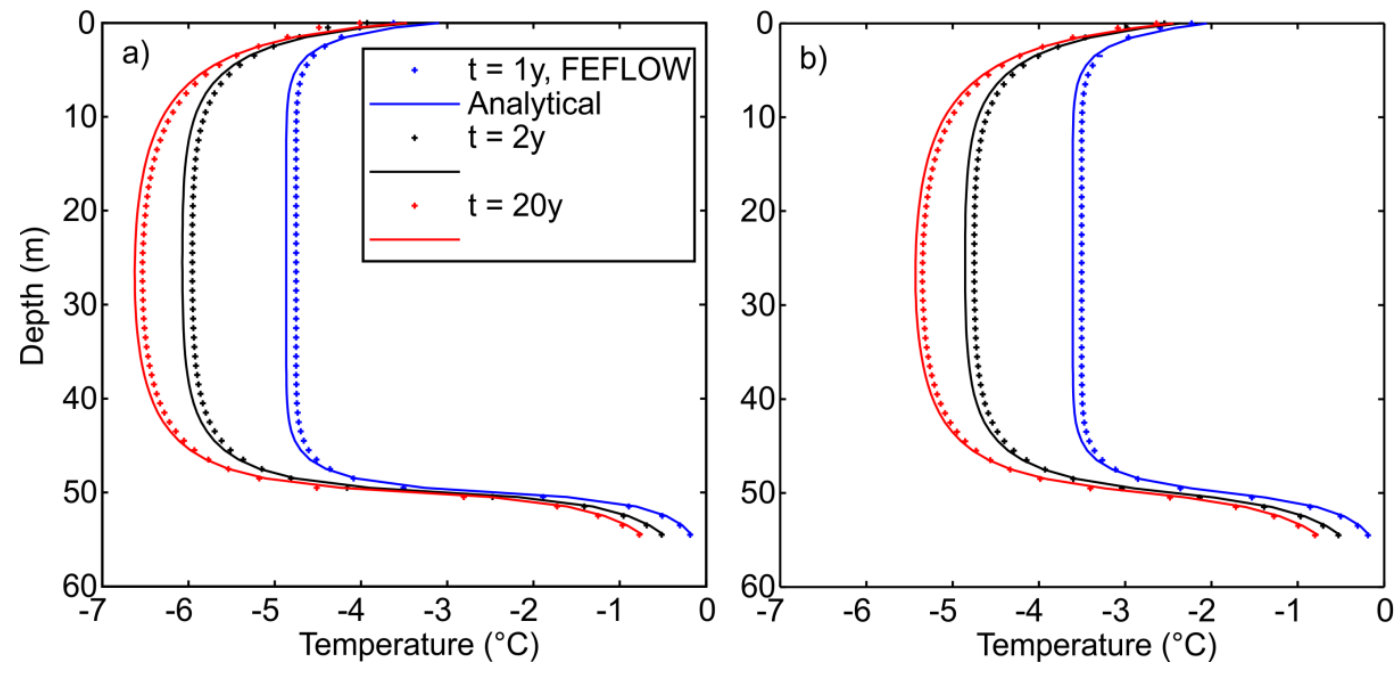

Figure 3: Temperature-depth profiles (TDPs) for synthetic model calculated with FEFLOW (dots) and the new analytical model (lines) after an operation period of $t=1,2$ and 20 years, and (a) at $0.5 \mathrm{~m}$ and (b) $1 \mathrm{~m}$ distance from the BHE; Darcy flux is $D=1 \times 10^{-7} \mathrm{~m} / \mathrm{s}$ (along the $x$ direction), $H=50 \mathrm{~m}, q=-30 \mathrm{~W} \mathrm{~m}^{-1}$.

a)

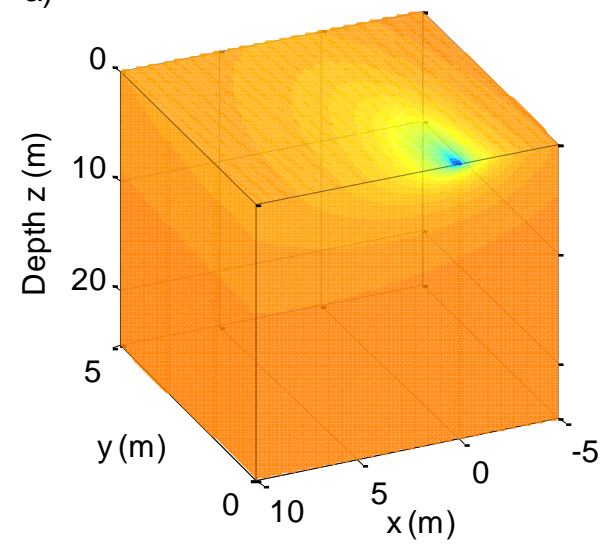

b)

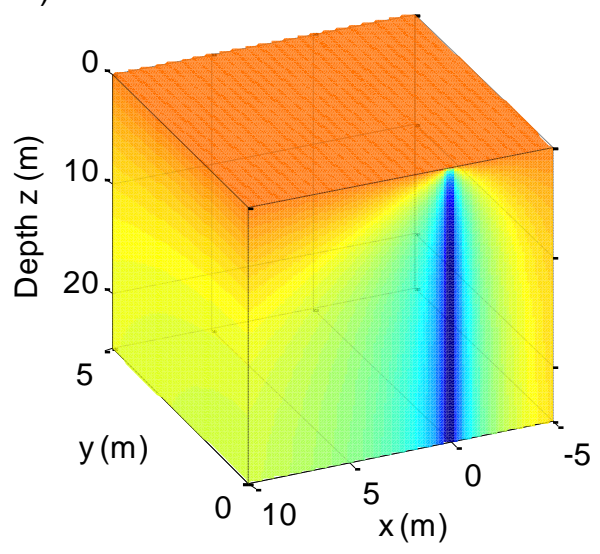

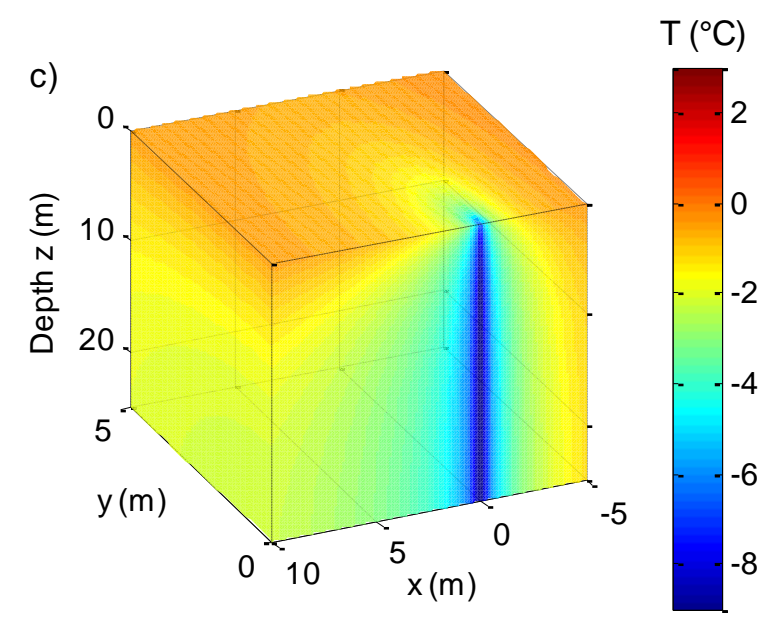

Figure 4: Temperature fields in the synthetic model after 20 years for the individual components in Eq. (4): (a) the Cauchy-type BC contribution, $\Delta T_{h}\left(\boldsymbol{x}, \boldsymbol{x}^{\prime}, t\right)$, (b) the standard $T_{M F L S}\left(\boldsymbol{x}, \boldsymbol{x}^{\prime}, t\right)$, and (c) the whole (superimposed) solution $T\left(\boldsymbol{x}, \boldsymbol{x}^{\prime}, t\right)$. Darcy flux is $D=1 \times 10^{-7} \mathrm{~m} / \mathrm{s}$ (along the $x$ direction); 
borehole length is $H=50 \mathrm{~m}, q=-30 \mathrm{~W} \mathrm{~m}^{-1}$ and the BHE is located at $(x=0, y=0, z=[0-50]) \mathrm{m}$. Only the top $25 \mathrm{~m}$ of the ground are shown.
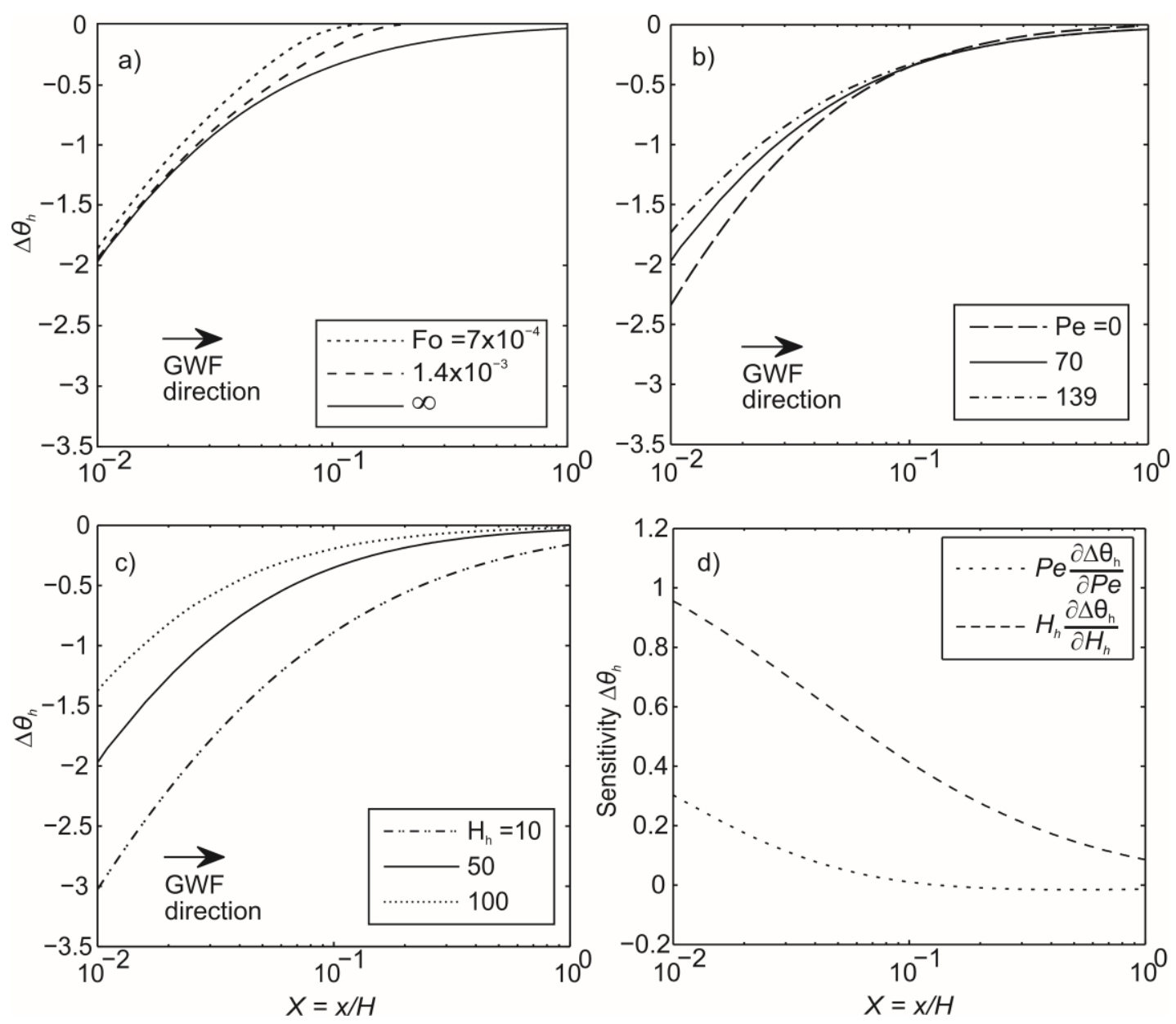

Figure 5: Dimensionless temperature at the ground surface $(y=0, z=0)$ along the normalized $x$ direction for different: (a) Fo numbers tuning the time $t,\left(P e=70, H_{h}=50\right)$ (b) $P e$ numbers changing the effective thermal velocity $v,\left(F o=\infty, H_{h}=50\right)$ (c) $H_{h}$ numbers modifying the surface heat exchange rate $h,(F o=\infty, P e=70)$, and (d) relative sensitivities with respect to $P e$ and $H_{h}$ numbers $\left(F o=\infty, P e=70, H_{h}=50\right)$. Groundwater flows along the $x$ direction (GWF). 

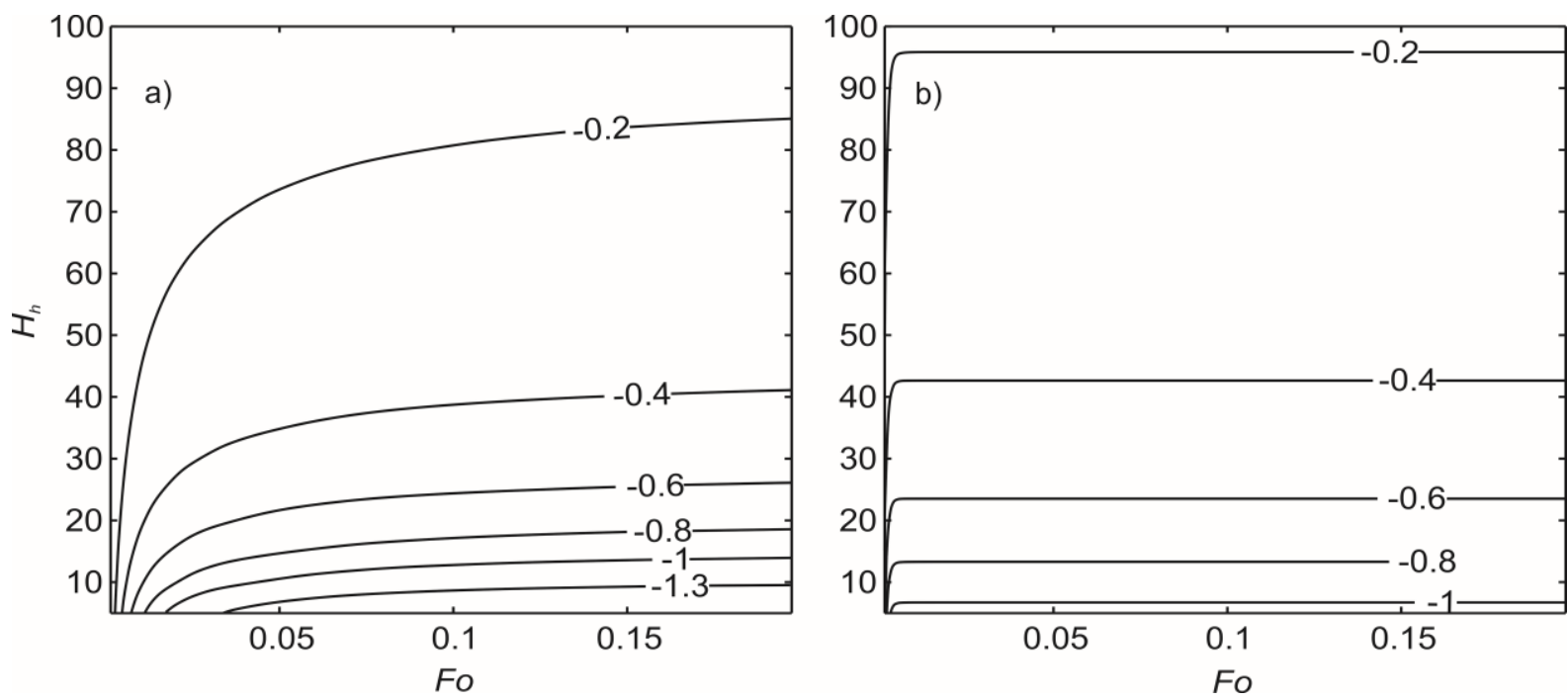

Figure 6: Dimensionless temperature $\Delta \theta_{h}$ at the ground surface $(x=0.1 H, y=0, z=0)$ for: (a) $P e=0$ and (b) $P e=70$. The BHE is located at $x=0 \mathrm{~m}$.
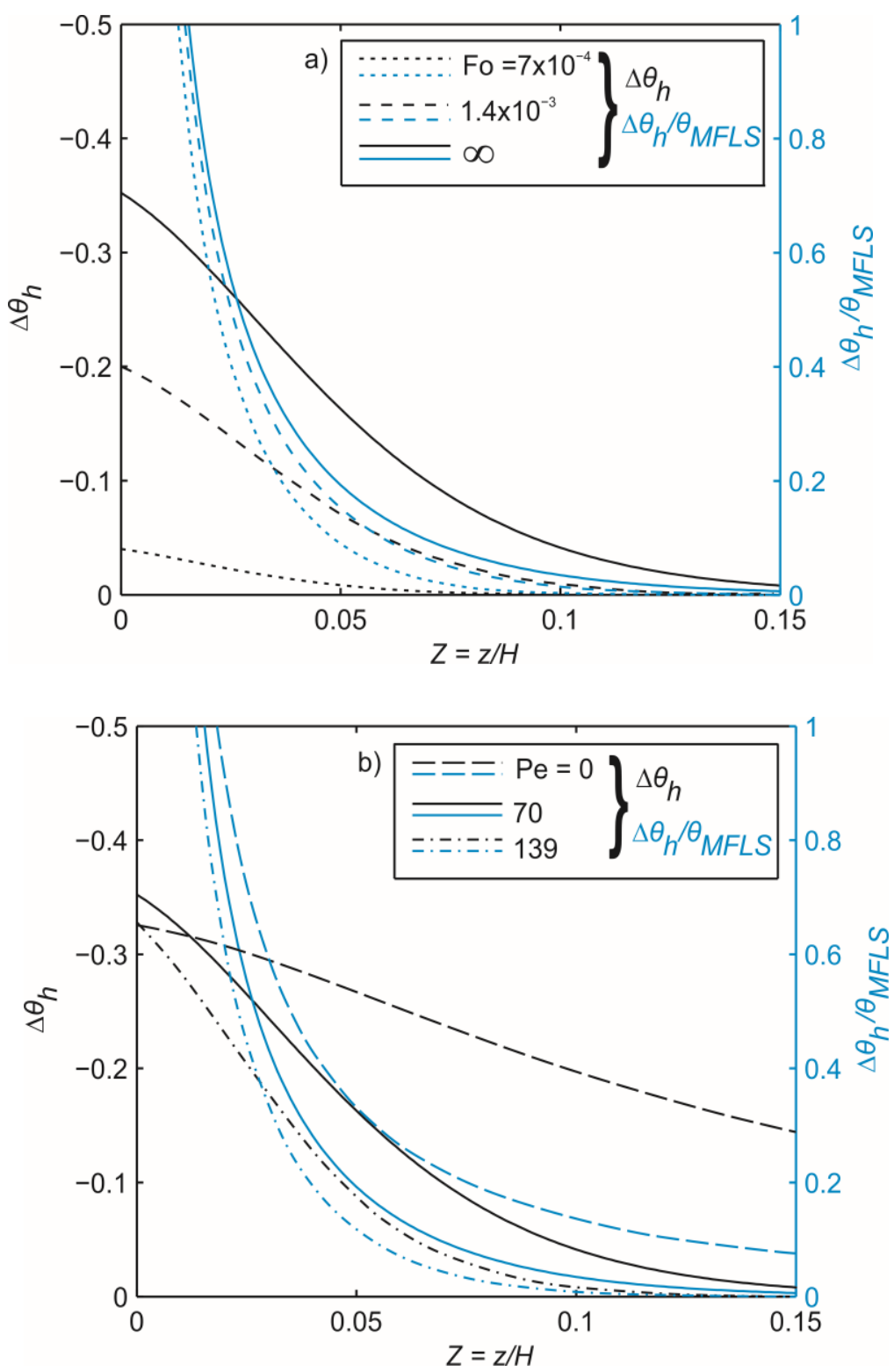


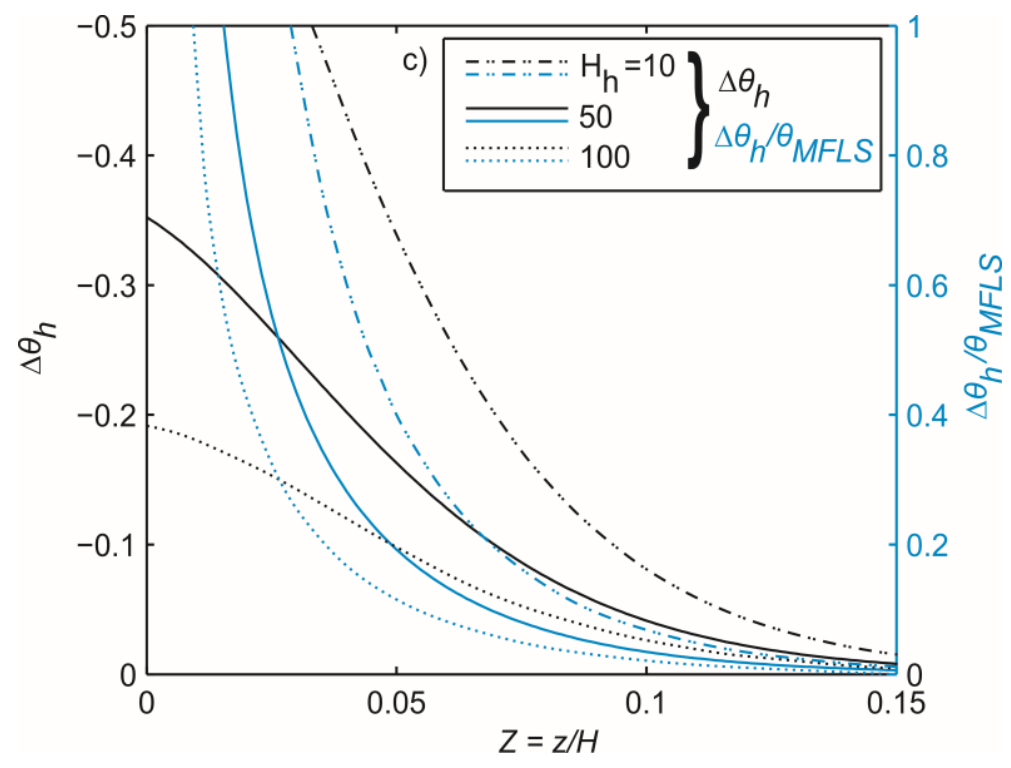

Figure 7: $\Delta \theta_{h}$ (black lines - left vertical axis) and ratio $\Delta \theta_{h} / \theta_{M F L S}$ (blue lines - right vertical axis) at $y=0, x=0.1 \mathrm{H}$ along the normalized $z$ direction for different: (a) $F o$ numbers changing the time $t,\left(P e=70, H_{h}=50\right)$ (b) $P e$ numbers changing the effective thermal velocity $v,(F o$ $\left.=\infty, H_{h}=50\right)$ and (c) $H_{h}$ numbers changing the surface heat exchange rate $h,(F o=\infty, P e=$ 70).

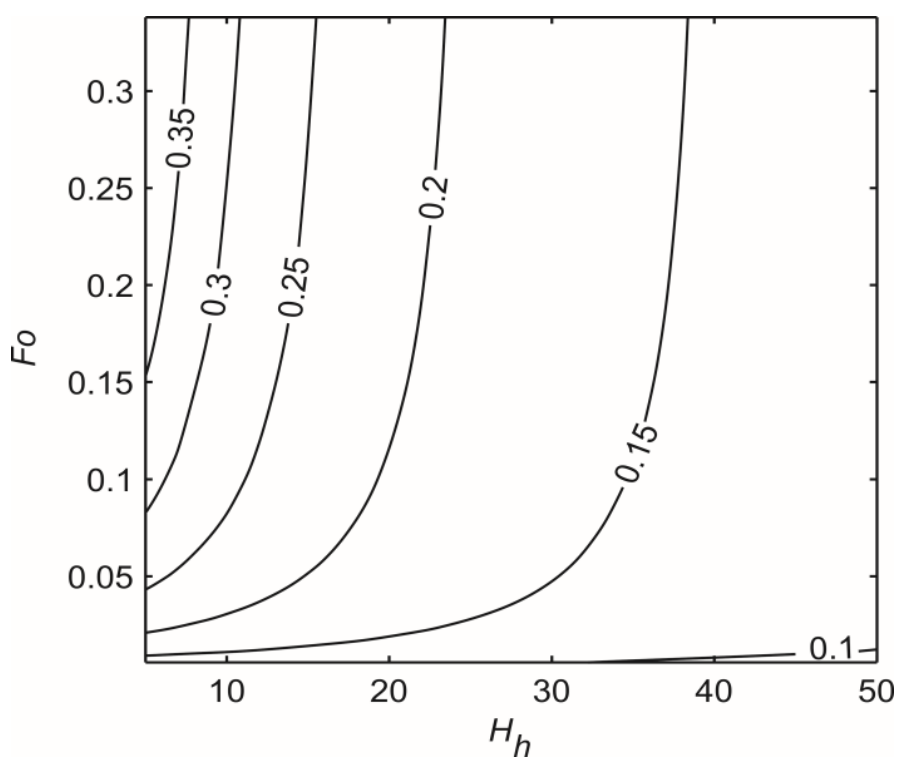

Figure 8: Maximum normalized depth $Z=z / H$ for $\Delta \theta_{h} / \theta_{M F L S} \geq 0.1$ at $x=0.1 H$ and $y=0$ for shallow BHEs under negligible groundwater flow. 


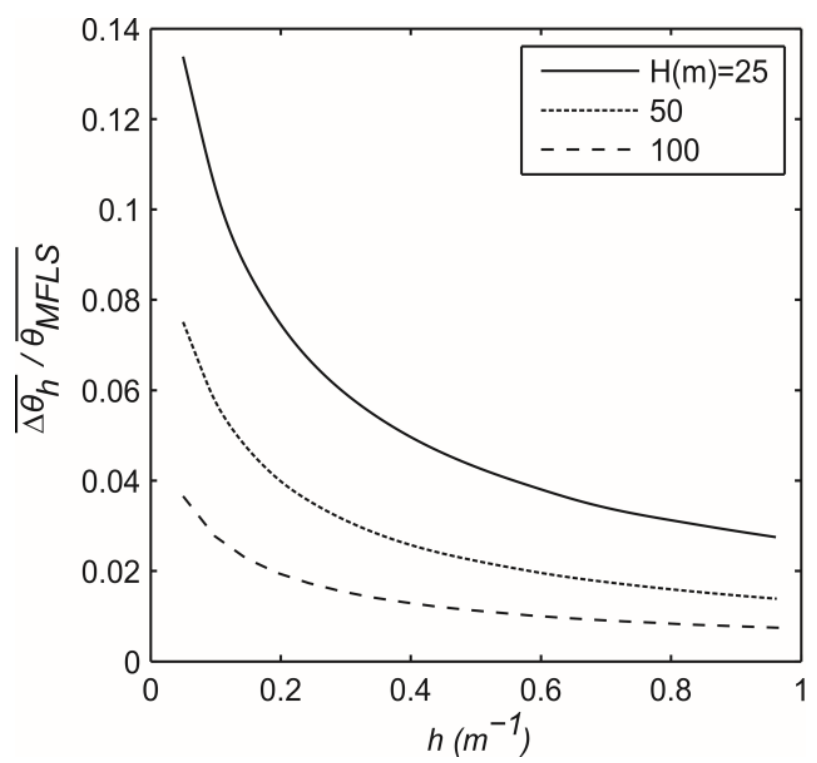

Figure 9: Ratio $\overline{\Delta \theta_{h}} / \overline{\theta_{M F L S}}$ for $r_{b}=0.1 \mathrm{~m}, P e=0$ and after 30 years of operation.

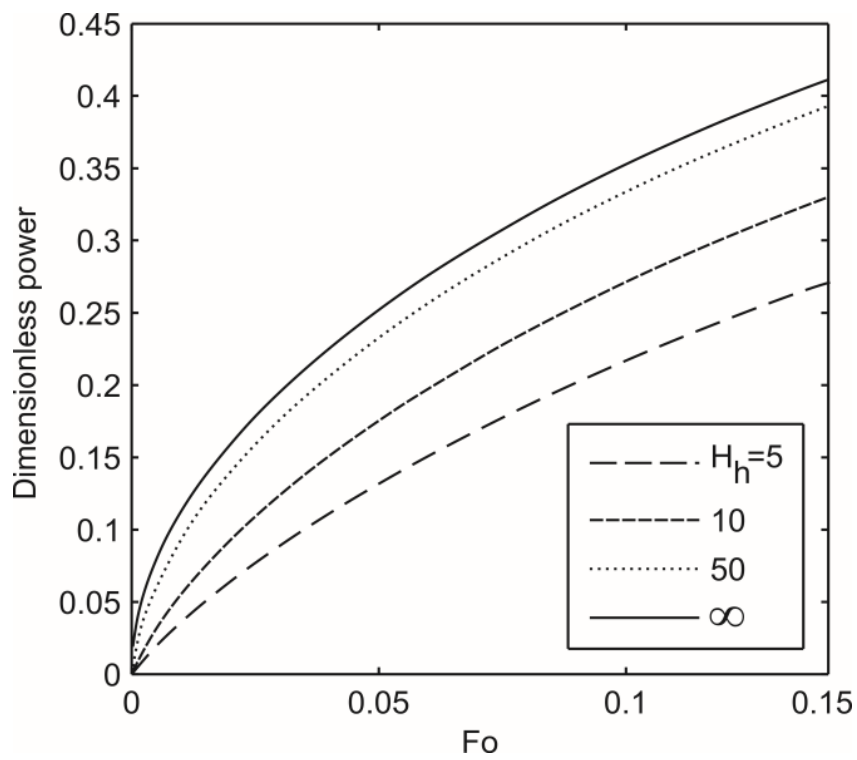

Figure 10: Dimensionless total power supply from the ground surface in dimensionless time, Fo. 


\section{Nomenclature}

$a$

$c$

Fo

G

$h$

$H$

$H_{h}$

$I$

$I_{0}$

$n_{\mathrm{e}}$

$p$

$P$

$P e$

D

$q$

$r_{b}$

$r_{d}$

$R$

$R_{b}$

$t$

$T$

$T_{o}$ thermal diffusivity $\left(\mathrm{m}^{2} \mathrm{~s}^{-1}\right)$

volumetric heat capacity $\left(\mathrm{MJ} \mathrm{m}^{-3} \mathrm{~K}^{-1}\right)$

Fourier number

Green's function

coupling coefficient $\left(\mathrm{m}^{-1}\right)$

borehole length (m)

dimensionless product $H \cdot h$

linear heat transfer coefficient $\left(\mathrm{W} \mathrm{m}^{-2} \mathrm{~K}^{-1}\right)$

modified Bessel function of first kind and of order zero

effective porous medium porosity

power (W)

dimensionless form of $p$

Péclet number

Darcy velocity $\left(\mathrm{m} \mathrm{y}^{-1}\right)$

heat flow rate per unit length $\left(\mathrm{W} \mathrm{m}^{-1}\right)$

borehole radius (m)

horizontal radial distance from the borehole (m)

dimensionless form of $r_{d}$

dimensionless form of $r_{b}$

time (s)

temperature in the porous medium $\left({ }^{\circ} \mathrm{C}\right)$

reference temperature $\left({ }^{\circ} \mathrm{C}\right)$ 


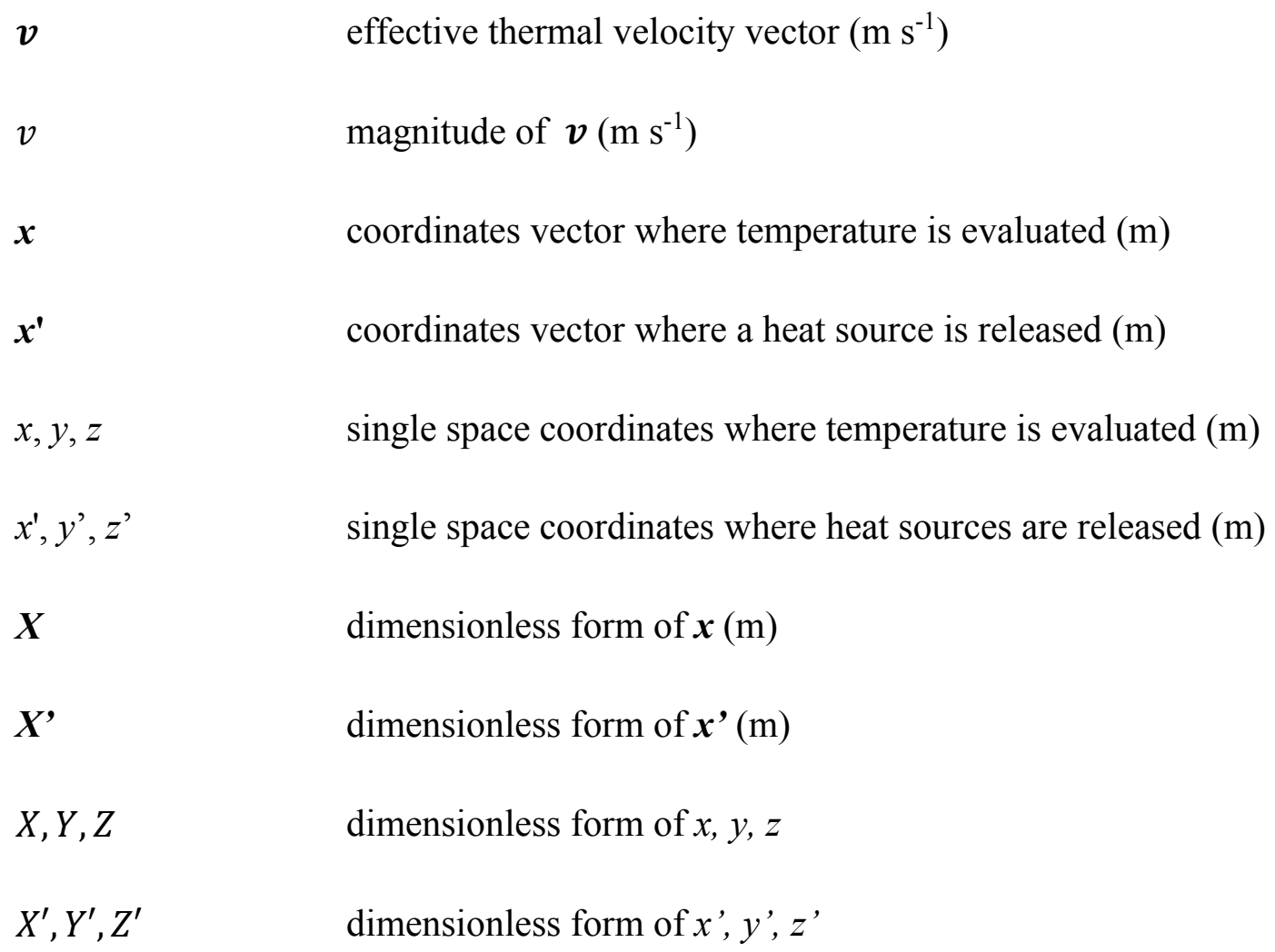

dimensionless form of $\boldsymbol{x}(\mathrm{m})$

dimensionless form of $\boldsymbol{x}^{\prime}(\mathrm{m})$

dimensionless form of $x, y, z$

Greek symbols

$\lambda$

$\kappa, \psi$

$\mathrm{K}, \mathrm{M}, \Psi$

$\tau$

$\theta$

$\varphi, \epsilon, \mu, \delta$

\section{Subscripts}

$\kappa, \mathrm{K}$

$h$

$w$

$S$

Abbreviations

substitution functions

intermediate or substitution variables

expressed in terms of the functions $\kappa, \mathrm{K}$

thermal conductivity $\left(\mathrm{W} \mathrm{m}^{-1} \mathrm{~K}^{-1}\right)$

dimensionless form of $\kappa, \mu, \psi$

time at which a heat pulse is released (s)

dimensionless temperature

referring to Cauchy-type boundary condition

wetting phase

ground surface 
$\mathrm{BC}$

BHE

GWF

GSHP

GST

MFLS

SAT

TDP

Other conventions

$\bar{x}$ boundary condition

borehole heat exchanger

groundwater flow

ground source heat pump

ground surface temperature

moving finite line source

surface air temperature

temperature depth profile

depth-averaged value of the quantity $x$ 


\section{Appendix C:}

With the dimensionless numbers identified in Eq. (9), the following are the normalized forms of relevant equations:

\begin{tabular}{|c|c|c|}
\hline $\begin{array}{l}\text { Equation } \\
\text { in the } \\
\text { main text }\end{array}$ & Dimensionless form & $\begin{array}{c}\text { Equation } \\
\text { id }\end{array}$ \\
\hline (5) & $\begin{aligned} \Delta \theta_{h}\left(\boldsymbol{X}, \boldsymbol{X}^{\prime}, P e, F o, H_{h}\right)=\Delta T_{h} \frac{4 \lambda \pi}{q} \\
=\exp \left(\frac{X P e}{2}\right) \int_{R^{2}}^{\infty} \frac{1}{\varphi} \exp (-\varphi \\
\left.-\left(\frac{R P e}{4}\right)^{2} \frac{1}{\varphi}\right)\left\{\exp \left(H_{h} Z\right.\right. \\
\left.+\left(\frac{H_{h} R}{2}\right)^{2} \frac{1}{\varphi}\right)\left[\operatorname{erfc}\left(\frac{Z \sqrt{\varphi}}{R}+\frac{H_{h} R}{2 \sqrt{\varphi}}\right)\right. \\
\left.\left.-\exp \left(H_{h}\right) \operatorname{erfc}\left(\frac{Z+1}{R} \sqrt{\varphi}+\frac{H_{h} R}{2 \sqrt{\varphi}}\right)\right]\right\} d \varphi\end{aligned}$ & $\mathrm{C} 1$ \\
\hline (6) & $\begin{aligned} \mathrm{K}\left(H_{h}, \mathrm{M}, Z, R\right) & =\kappa\left(h, \mu, z, r_{d}\right) \frac{H}{H} \\
& =\frac{2}{R} \sqrt{\frac{\varphi}{\pi}} \int_{0}^{\infty} \exp \left[-H_{h} \mathrm{E}-\varphi\left(\frac{Z+\mathrm{M}+\mathrm{E}}{R}\right)^{2}\right] d \mathrm{E}\end{aligned}$ & $\mathrm{C} 2$ \\
\hline (7) & $\begin{aligned} \Delta \theta_{h \kappa}=\Delta T_{h \kappa} \frac{4 \lambda \pi}{q} & \\
& =\exp \left(\frac{X P e}{2}\right) \int_{\frac{R^{2}}{4 F o}}^{\infty} \frac{1}{\varphi} \exp (-\varphi \\
& \left.-\left(\frac{R P e}{4}\right)^{2} \frac{1}{\varphi}\right)\left[\mathrm{K}\left(H_{h}, 0, Z, R\right)-\mathrm{K}\left(H_{h}, 1, Z, R\right)\right] d \varphi\end{aligned}$ & $\mathrm{C} 3$ \\
\hline (13) & $\begin{aligned} \overline{\Delta \theta_{h}}=\overline{\Delta T_{h}} \frac{4 \lambda \pi}{q} & =\frac{1}{H_{h}} I_{0}\left(\frac{R_{b} P e}{2}\right) \int_{\frac{R_{b}{ }^{2}}{4 F o}}^{\infty} \frac{1}{\varphi} \exp (-\varphi \\
& \left.-\left(\frac{R_{b} P e}{4}\right)^{2} \frac{1}{\varphi}\right)\left[\Psi\left(H_{h}, R_{b}, \varphi\right)\right] d \varphi\end{aligned}$ & $\mathrm{C} 4$ \\
\hline (14) & $\begin{array}{l}\Psi\left(H_{h}, R_{b}, \varphi\right)=2 \operatorname{erf}\left(\frac{1}{R_{b}} \sqrt{\varphi}\right)-\operatorname{erf}\left(\frac{2}{R_{b}} \sqrt{\varphi}\right)+ \\
\exp \left[\left(\frac{H_{h} R_{b}}{2}\right)^{2} \frac{1}{\varphi}\right]\left[\exp \left(H_{h}\right) \operatorname{erfc}\left(\frac{1}{R_{b}} \sqrt{\varphi}+\frac{H_{h} R_{b}}{2 \sqrt{\varphi}}\right)-\operatorname{erfc}\left(\frac{H_{h} R_{b}}{2 \sqrt{\varphi}}\right)-\right.\end{array}$ & $\mathrm{C} 5$ \\
\hline
\end{tabular}




\begin{tabular}{|c|r|r|}
\hline & $\left.\exp \left(2 H_{h}\right) \operatorname{erfc}\left(\frac{2}{R_{b}} \sqrt{\varphi}+\frac{H_{h} R_{b}}{2 \sqrt{\varphi}}\right)+\exp \left(H_{h}\right) \operatorname{erfc}\left(\frac{H}{R_{b}} \sqrt{\varphi}+\frac{H_{h} R_{b}}{2 \sqrt{\varphi}}\right)\right]$ & \\
\hline (15) & $\Psi_{\mathrm{K}}\left(H_{h}, R_{b}, \varphi\right)=2 \operatorname{erf}\left(\frac{1}{R_{b}} \sqrt{\varphi}\right)-\operatorname{erf}\left(\frac{2}{R_{b}} \sqrt{\varphi}\right)+\mathrm{K}\left(H_{h}, 1,0, R_{b}\right)$ & $\mathrm{C} 6$ \\
& $-\mathrm{K}\left(H_{h}, 0,0, R_{b}\right)-\mathrm{K}\left(H_{h}, 1,1, R_{b}\right)+\mathrm{K}\left(H_{h}, 0,1, R_{b}\right)$ & \\
\hline
\end{tabular}

